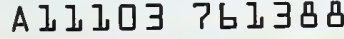

NIST

PUBLICATIONS
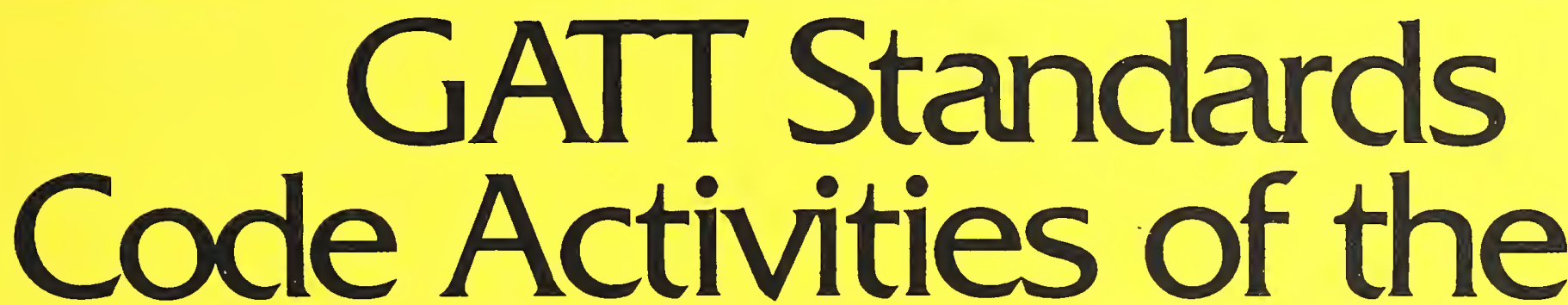

National Institute of
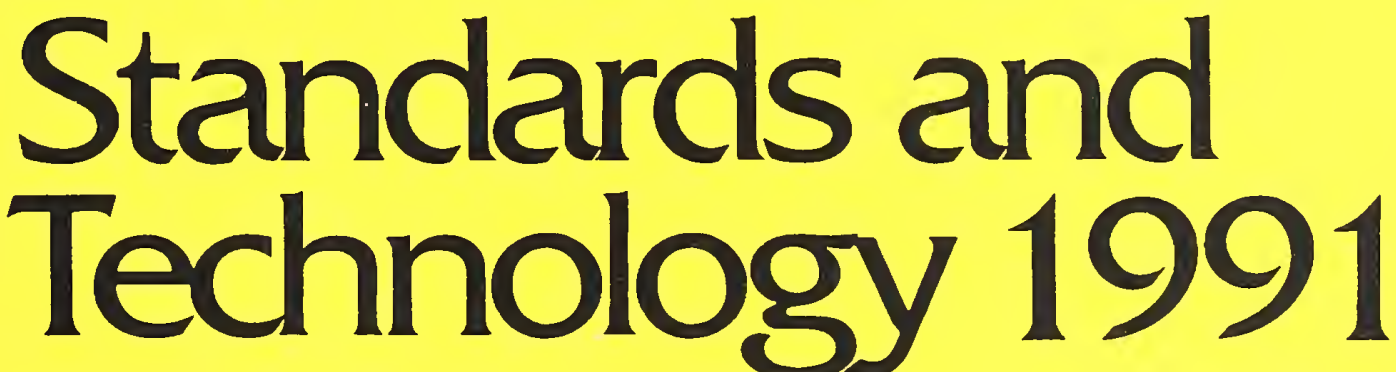

JoAnne R. Overman

Office of Standards Services

$-\mathrm{QC}$

100

.456

4829

1992

C. 2 D United States Department of Commerce

National Institute of Standards and Technology

April 1992 



\title{
GATT STANDARDS CODE ACTIVITIES OF THE NATIONAL INSTITUTE OF STANDARDS AND TECHNOLOGY 1991
}

\author{
JoAnne R. Overman
}

U.S. DEPARTMENT OF COMMERCE Technology Administration National Institute of Standards and Technology

Standards Code and Information Program Office of Standards Services

Gaithersburg, MD 20899

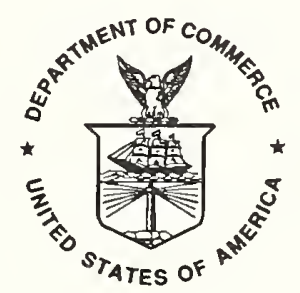

U.S. DEPARTMENT OF COMMERCE Barbara Hackman Franklin, Secretary

TECHNOLOGY ADMINISTRATION

Robert M. White, Under Secretary for Technology

NATIONAL INSTITUTE OF STANDARDS

AND TECHNOLOGY

John W. Lyons, Director 



\section{ABSTRACT}

This report describes the GATT standards code activities conducted by the standards Code and Information Program, National Institute of standards and Technology (NIST), for calendar year 1991. NIST responsibilities include operating the U.S. GATT inquiry point for information on standards and certification activities: notifying the GATT Secretariat of proposed U.S. Federal Government standards-based rules that might significantly affect trade; assisting U.S. industry with standards-related trade problems; and responding to inquiries about proposed foreign and U.S. regulations.

Key Words: certification information; GATT standards Code; notifications; proposed foreign regulations; standards information; technical assistance 


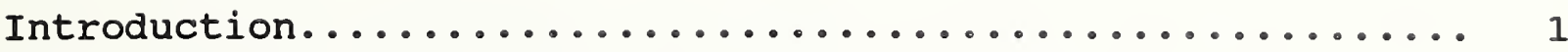

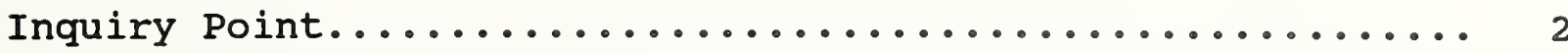

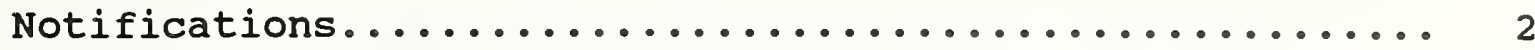

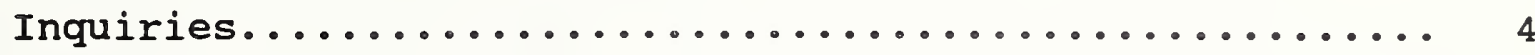

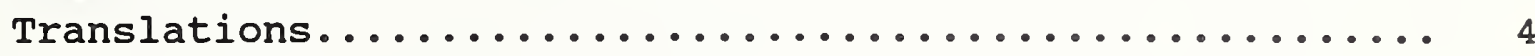

Standards Information......................... 4

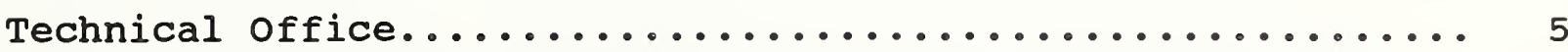

Comment Handling.......................... 6

Comment Periods............................. 6

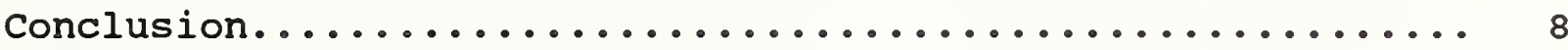

Tables

Table I Standards Code signatories ................ 9

Table II Number of Notifications Issued in 1991...... 10

Table III List of U.S. Notifications.............. 11

Table IV List of Foreign Notifications............ 13

Appendix A - Key-Word-Out-of-Context (KWOC) Index of

Products Notified........................... 21

Appendix B - Number of Inquiries by signatories ......... 45 

The Agreement on Technical Barriers to Trade (popularly known as the standards code) was negotiated and accepted during the Tokyo Round of Multilateral Trade Negotiations in the 1970's. The Standards code is designed to eliminate the use of standards, technical regulations, and conformity assessment (certification) procedures as unnecessary barriers to trade. The trade negotiations are held under the auspices of the General Agreement on Tariffs and Trade (GATT). As this report goes to press the Contracting Parties participating in the current Uruguay Round are still discussing various issues and hope to conclude the negotiations, which will result in a revised standards code, some time this year.

There are currently 40 signatories to the Standards code. Thirty of the signatories have actively implemented the code in their countries. The other 10 signatories have either not: 1) ratified the Agreement; 2) established an inquiry point for standards information; 3) reported any proposed government mandatory regulations (see Table I); or 4) some combination of the foregoing. The code is administered by the GATT Secretariat in Geneva, Switzerland.

After the conclusion of the Tokyo Round, the Trade Agreements Act of 1979 ratified the United States' accession to the GATT Standards Code. The NIST Office of Standards Services (OSS) is responsible for several provisions which relate to the establishment of a U.S. inquiry point, ${ }^{1}$ a standards information center, and a technical office for non-agricultural products.

This report summarizes standards code activities conducted by the standards code and Information (SCI) Program for calendar year 1991.

1 The Standards code requires that each signatory provide an inquiry point to answer all requests for information about technical regulations, standards, and rules of conformity assessment. 


\section{Notifications}

Signatories of the Standards code must notify the GATT Secretariat of all proposed government regulations and conformity assessment procedures that might significantly affect international trade. The secretariat, in turn, disseminates the notifications to all other signatories. As the official U.S. GATT inquiry point for information on standards and certification activities, SCI notifies the Secretariat of proposed U.S. Federal Government rules that might significantly affect trade. SCI also receives foreign notifications through the GATT secretariat and disseminates them, along with supplementary information, to appropriate U.S. Companies and industry groups. SCI responds to inquiries about foreign and U.S. notifications, provides copies of the proposed regulations, and arranges for translations of foreign standards. SCI also assists in developing comments on the impact on U.S. industry of proposed foreign regulations and transmits them to the foreign government concerned.

In 1991 the GATT Secretariat issued 358 notifications. Twenty-six signatories reported at least one proposed regulation. (See Table II.) Japan had the most notifications (49). Fourteen other signatories - Austria, Belgium, Canada, Czechoslovakia, Denmark, EC, Finland, New Zealand, Norway, Philippines, Sweden, Switzerland, the United States and Yugoslavia - each reported 10 or more proposed regulations.

An official in the office of the U.S. Trade Representative (USTR), based in Geneva, receives notifications from the GATT secretariat and cables them to the United States. The notifications, all of which are available in English, include the name of the country proposing the regulation; the assigned TBT/Notification number; a brief description of the regulation; and the final date for comments. In order to provide wide access to this information in the United States, all foreign notifications are published in the Commerce Business Daily and several other U.S. publications. SCI maintains a computerized database on all GATT notifications and a telephone GATT hotline where up-to-date notification information can be obtained. The hotline number is (301) 975-4041 (not toll free).

Proposed U.S. Regulations

All proposed U.S. Government rules (mandatory standards or conformity assessment systems), including proposed revisions, are published in the Federal Register by the responsible Federal agency. SCI staff regularly review the Register to identify those proposed regulations that should be notified to the GATT Secretariat and send notices directly to the GATT Secretariat via telefax. 
In 1991, 45 proposed U.S. regulations, covering such subjects as food labeling, motor vehicles, drugs and distilled spirits were reported. The originating agencies are shown below. (See Table III for listing of specific products by agency.)

\section{Agency}

No. of 1991 Notifs.

Bureau of Alcohol, Tobacco \& Firearms (BATF)

Consumer Product Safety Commission (CPSC)

Dept. of Commerce (DOC)

Dept. of Treasury (DOT)

Environmental Protection Agency (EPA)

Food \& Drug Administration (FDA)

National Highway Traffic Safety Admin. (NHTSA)

U.S. Dept. of Agriculture (USDA)

$\begin{array}{rr} & 5 \\ & 4 \\ & 1 \\ & 1 \\ & 1 \\ & 9 \\ & 20 \\ & 4 \\ \text { Total } & 45\end{array}$

Proposed Foreign Regulations

The GATT Secretariat in 1991 issued 313 notifications from countries other than the United States. (See Table IV.) The foreign regulations covered a wide variety of products, including household appliances, food, ozone-depleting substances, plants, breath testing apparatus, and gondolas. SCI maintains a Key-Wordout-of-Context (KWOC) index for all products that are the subject of foreign notifications. (See Appendix A for the 1991 index.) (KWOC is an indexing method in which keywords, in this instance product names, appear as headings on a separate line.) The KWOC index is used to identify regulations that may be of interest to individuals who have expressed interest in specific products.

SCI regularly sends foreign notifications to members of the Industry Functional Advisory Committee on Standards (IFAC) ${ }^{2}$ and several other groups and specific individuals to serve their information needs and to allow them to disseminate this information to others. SCI sends copies of all foreign notifications to the American National Standards Institute for subsequent publication in its biweekly ANSI Reporter.

2 The IFAC is comprised of Government-appointed individuals from private industry engaged in standards-related activities. It advises the Department of Commerce and USTR on trade matters, the operation of any adopted trade agreements, and other subjects related to U.S. trade policy. 


\section{Inquiries}

SCI responded to 400 domestic and foreign letter and telephone requests for GATT notification information (277 U.S., 123

foreign) in 1991. Many requestors asked for copies of more than one regulation. In addition a number of requestors asked for general information on the standards Code. SCI responds to such requests by sending an information package consisting of the latest issue of: its newsletter, TBT News; a list of recent foreign notifications; brochures describing the standards Code; and ordering information for the Commerce Business Daily.

In 1991, the most often requested regulations concerned medical devices, terminal equipment, accreditation systems, chemicals which may harm the environment, and timber/lumber. In addition, 501 individuals called the GATT hotline for up-to-date information on foreign notifications.

\section{Translations}

SCI requests the text of all proposed foreign regulations which are notified. Signatory countries are required to supply copies of the regulations through their inquiry points. Of the 297 regulations received in 1991, 107 were in languages other than English. SCI coordinates a service to obtain, on request, an English translation of any proposed regulation in a foreign language. The cost of translation is paid by the requestor; if more than one request is received for the same translation, the cost is divided equally among requestors. In 1991, SCI received five requests for translation of five regulations and standardsrelated documents. The cost of a translation is determined by the number of words contained in the document and the price per 1,000 words charged by the contractor.

\section{STANDARDS INFORMATION}

The National Center for Standards and Certification Information (NCSCI) is the national focal point for standards-related information. NCSCI supports GATT inquiry point activities by supplying information concerning both domestic and foreign standards. The information center, established in 1965, provides government, industry, and the public with information on standards, regulations, certification programs, and related activities that affect trade and commerce.

NCSCI maintains a microform and/or hard copy collection of engineering standards and specifications, test methods, regulations, certification rules, directories, reference books, and special publications. In 1991, NCSCI staff responded to more than 6,500 requests from all over the world on the existence, source, and availability of standards and related documents. Thirty of the 40 signatories contacted the center for 
information. (See Appendix B.) NCSCI also responds to trade-related queries regarding regulations or other requirements imposed by foreign countries that affect the export of U.S. manufactured products.

In 1991, SCI published and NCSCI distributed the following reports:

- Laboratory Accreditation in the United States (NISTIR 4576);

- Questions and Answers on Quality, the ISO 9000 standard Series, Quality system Registration and Related Issues (NISTIR 4721);

- Standards Activities of Organizations in the United States (NIST SP 806);

- Directory of Federal Government Laboratory Accreditation Designation Programs (NIST SP 808); and

- Directory of State and Local Government Laboratory Accreditation/Designation Programs (NIST SP 815).

In a related area - the EC 1992 single Internal Market - NCSCI maintains an EC hotline to report on draft CEN and CENELEC standards and EC directives related to standards which may affect trade. The recorded hotline - (301) 921-4164 (not toll-free) is updated weekly and provides the subject/product areas, document number, and final date for comments. A total of 2,087 calls were received on the hotline during 1991.

NCSCI staff also review the official Journal of the European Communities to identify proposed standards-related directives. Copies of the directives are sent to the appropriate trade or professional associations for dissemination to and review by their members.

\section{TECHNICAL OFFICE}

The Technical office, established by the Trade Agreements Act of 1979, is responsible for providing technical support related to non-agricultural products. Office functions include:

a) participation in standards-related conferences; b) technical assistance to the International Trade Administration of the Department of Commerce and the office of the U.S. Trade Representative in developing proposals to enhance the standards Code; c) preparation of special reports on standards issues; and d) coordination of comments on proposed foreign regulations.

1991 Technical office activities included:

- Continued participation by office staff in tri-lateral negotiations with Mexican and Canadian officials to establish the terms of the standards obligations of the North America Free Trade Agreement. 
- Continued work with other Government agencies to inform U.S. industry of EC 1992 standards-related activities. These activities included participating in the EC 1992 Work Group on Standards, Testing, and Certification and the U.S./EC Study Group concerning the Joint Communique signed by former commerce Secretary Robert A. Mosbacher and EC Vice President Martin Bangemann, and making presentations on EC 1992 standards development activities to interested U.S. industry groups.

\section{Comment Handling}

A major objective of the standards code is to allow exporters, government agencies, and other interested parties to comment on proposed foreign regulations which they believe would unjustifiably impede exports. SCI provides the text of proposed foreign regulations on request and encourages the submission of comments if a regulation appears to create unnecessary trade barriers. SCI transmits comments to the appropriate foreign government and coordinates any follow-up activity. In 1991, SCI received and processed formal comments from U.S. industry on three foreign regulations: Sweden (91.86) - products containing mercury; Denmark (91.205) - nickel containing products; and Sweden $(90.317)$ - cosmetics.

Additional informal comments are frequently made by industry representatives who become aware of proposed regulations, often even before they are officially notified through the GATT. The notification system can often serve as a back-up mechanism to ensure awareness of foreign developments.

\section{Comment Periods}

So that signatories will have adequate time to receive the text of a regulation (and to have it translated if necessary), review it, and prepare comments, the standards code recommends a comment period of at least 60 days. If the comment period is insufficient (e.g., due to late receipt of an English version or complicated technical issues), the SCI staff will request that the issuing country extend the comment period. In 1991, six such requests were made by the United States to the notifying Parties. Four of the extension requests were granted. Many extensions are requested so that individuals can translate and study the regulation without necessarily indicating an intention to comment. SCI staff also received seven requests from other signatories requesting extensions of the comment period for proposed U.S. technical regulations. The comment period for two of the regulations were officially extended. 
The average length of the comment period ${ }^{3}$ in 1991 was 46 days for U.S. regulations and 43 days for foreign regulations. "The average length of the comment period, by U.S. agency, is shown below:

$\begin{array}{ll}\text { BATF } & 49 \text { days } \\ \text { CPSC } & 52 \text { days } \\ \text { DOC } & 34 \text { days } \\ \text { DOT } & 18 \text { days } \\ \text { EPA } & 34 \text { days } \\ \text { FDA } & 39 \text { days } \\ \text { NHTSA } & 43 \text { days } \\ \text { USDA } & 59 \text { days }\end{array}$

Comment periods for foreign regulations ranged from none at all to 215 days. In instances where there was no comment period provided, the average length is "0". The average length of comment period by country was:

$\begin{array}{lrlr}\text { Austria } & 82 \text { days } & \text { Ireland } & 0 \text { days } \\ \text { Belgium } & 0 \text { days } & \text { Japan } & 63 \text { days } \\ \text { Brazil } & 0 \text { days } & \text { Korea } & 43 \text { days } \\ \text { Canada } & 23 \text { days } & \text { Netherlands } & 49 \text { days } \\ \text { Czechoslovakia } & 22 \text { days } & \text { New Zealand } & 54 \text { days } \\ \text { Denmark } & 26 \text { days } & \text { Norway } & 69 \text { days } \\ \text { EC } & 49 \text { days } & \text { Philippines } & 9 \text { days } \\ \text { Finland } & 71 \text { days } & \text { Singapore } & 48 \text { days } \\ \text { France } & 0 \text { days } & \text { Sweden } & 35 \text { days } \\ \text { Germany } & 17 \text { days } & \text { Switzerland } & 53 \text { days } \\ \text { Hong Kong } & 33 \text { days } & \text { United Kingdom } & 33 \text { days } \\ \text { Hungary } & 14 \text { days } & \text { Yugoslavia } & 58 \text { days } \\ \text { India } & 10 \text { days } & & \end{array}$

3 Number of calendar days between the date the notification was issued by the GATT secretariat and the closing date for comments. 


\section{CONCLUSION}

SCI continues to work with U.S. industry to address standardsrelated trade problems and to assure wide distribution of relevant notifications of proposed foreign regulations which might affect trade. Requests for further information, suggestions on how the service might be made more useful, or comments on subjects covered in this report should be directed to:

Standards Code and Information Program National Institute of Standards and Technology TRF Building, Room Al63 Gaithersburg, MD 20899

$$
\text { FAX: (301) } \quad 926-1559
$$

For specific standards-related information from NCSCI, call (301) $975-4040,-4038$, or -4036 .

For up-to-date information on proposed foreign regulations, call the GATT Hotline (301) 975-4041.

For up-to-date information on draft CEN/CENELEC documents, call the EC Hotline (301) 921-4164. 


\section{Standards code Signatories}

Argentina (a)
Austria
Belgium
Brazil
Canada
Chile (b)
Czechoslovakia
Denmark
Egypt (b)
European Community (EC)
Finland
France
Federal Republic of
Germany
Greece (b)
Hong Kong
Hungary
India
Ireland
Israel (b)
Italy

Japan

Korea

Luxembourg

(b)

Mexico (b)

Netherlands

New Zealand

Norway

Pakistan (b)

Philippines

Portugal (b)

Romania

Rwanda (a) (c)

Singapore

Spain

Sweden

Switzerland

Tunisia

United Kingdom

United States

Italy

Yugoslavia

(a) - the country has not yet ratified the standards code and has not reported any proposed regulations to the GATT secretariat.

(b) - the country has not reported any proposed regulations to the GATT Secretariat.

(c) - the country has not established an inquiry point for standards information and has not reported any proposed regulations to the GATT secretariat. 
Notifying Countries and

Number of GATT Notifications Issued in 1991

\section{Signatory}

Austria

Belgium

Brazil

Canada

Czechoslovakia

Denmark

EC

Finland

France

Germany

Hong Kong

Hungary

India

Ireland

Japan

Korea

Netherlands

New Zealand

Norway

Philippines

Singapore

Sweden

Switzerland

United Kingdom

United States

Yugoslavia
Number of

Notifications

13

10

1

35

12

13

14

27

2

9

5

2

6

1

49

9

4

11

13

19

4

24

16

2

45

12

Total 358 
TABLE III

List of U.S. Notifications

TBT/NDTIF。\#

$\begin{array}{ll}91.21 & \\ 91.39 & \text { USOA }(320) * \\ 91.42 & \text { FOA }(321) \\ 91.49 & \text { NHTSA }(322) \\ 91.62 & \text { NHTSA }(323) \\ 91.66 & \text { BATF }(324) \\ 91.68 & \text { FOA }(325) \\ 91.69 & \text { CPSC }(326) \\ 91.70 & \text { NHTSA }(328) \\ 91.81 & \text { NHTSA }(327) \\ 91.97 & \text { EPA }(329) \\ 91.98 & \text { NHTSA }(331) \\ 91.121 & \text { NHTSA }(330) \\ 91.122 & \text { NHTSA }(332) \\ 91.123 & \text { CPSC }(333) \\ 91.138 & \text { CPSC }(334) \\ 91.140 & \text { NHTSA }(335) \\ 91.141 & \text { BATF }(337) \\ 91.151 & \text { FOA }(336) \\ 91.152 & \text { NHTSA }(340) \\ 91.153 & \text { NHTSA }(339) \\ 91.185 & \text { USDA }(338) \\ 91.195 & \text { FDA }(341) \\ 91.202 & \text { FOA }(342) \\ 91.215 & \text { NHTSA }(343) \\ 91.216 & \text { FOA }(344) \\ 91.218 & \text { FOA }(345) \\ 91.226 & \text { NHTSA }(346) \\ 91.241 & \text { CPSC }(347) \\ 91.242 & \text { NHTSA }(349) \\ 91.243 & \text { NHTSA }(348) \\ 91.263 & \text { USOA }(350) \\ 91.267 & \text { NHTSA }(351) \\ 91.271 & \text { NHTSA }(352) \\ 91.281 & \text { BATF }(353) \\ 91.293 & \text { BATF }(354) \\ 91.294 & \text { NHTSA }(357) \\ 91.295 & \text { FOA }(356) \\ 91.301 & \text { BOT }(355) \\ 91.317 & \text { DOC }(358) \\ 91.330 & \text { FDA }(361) \\ 91.331 & \text { USOA }(360) \\ 91.333 & \text { NHTSA }(362) \\ 91.334 & \text { NHTSA }(363) \\ 91.342 & \\ 9 H T S A(364)\end{array}$

PROOUCT

HAM \& SAUSAGE

DIAGNOSTIC X-RAY SYSTEMS

MOTOR VEHICLE OISPLAYS \& CONTROLS

MOTOR VEHICLES \& EQUIPMENT

WINE CONTAINERS

ORUGS \& FINISHEO PHARMACEUTICALS

FIREWORKS

PASSENGER CAR TIRES

BRAKE HOSES

MOTOR VEHICLES

SCHOOL BUSES

SCHOOL BUS BOOY

TIRE SELECTION \& RIMS

ART MATERIALS

ART MATERIALS

GLAZING MATERIALS

VODKA

OVER-THE-CDUNTER DRUGS

AIR BRAKE SYSTEMS

AUTOMATIC BRAKE AOJUSTERS

PISTACHIO NUTS

YDGURT

FOOD LABELING

MDTORCYCLES

DILUTED JUICE BEVERAGES

RAW FRUIT, VEGETABLES, \& FISH

HYORAULIC BRAKE SYSTEMS

INFANT CUSHIONS \& PILLOWS

CHILD RESTRAINT SYSTEMS

LAMPS, REFLECTIVE DEVICES

MEAT \& MEAT PROOUCTS

LAMPS, REFLECTIVE DEVICES

SCHOOL BUSES

DISTILLED SPIRITS

WINE

LAMPS, REFLECTIVE DEVICES \& ASSOC. EQUIPMENT

STERILE PHARMACEUTICALS

IMPORTED MERCHANOISE

OISTILLEO SPIRITS

TOY, LOOK-ALIKE \& IMITATION FIREARMS

FOOD FOR HUMAN CONSUMPTION

MEAT \& POULTRY PROOUCTS

MOTOR VEHICLES

TRAILERS

LAP BELTS

*CONTROL NUMBER 

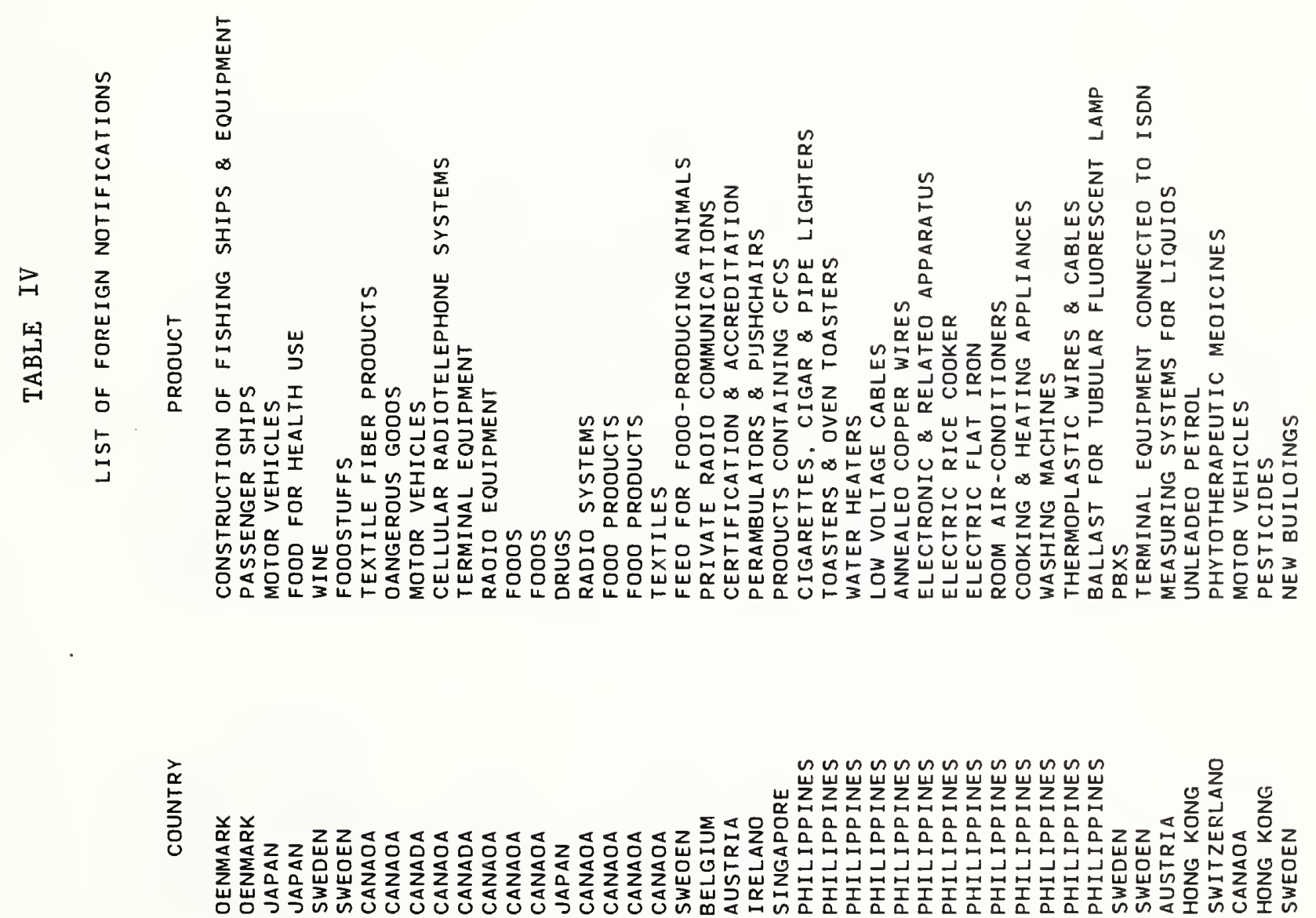
皮

-

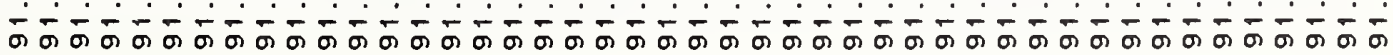



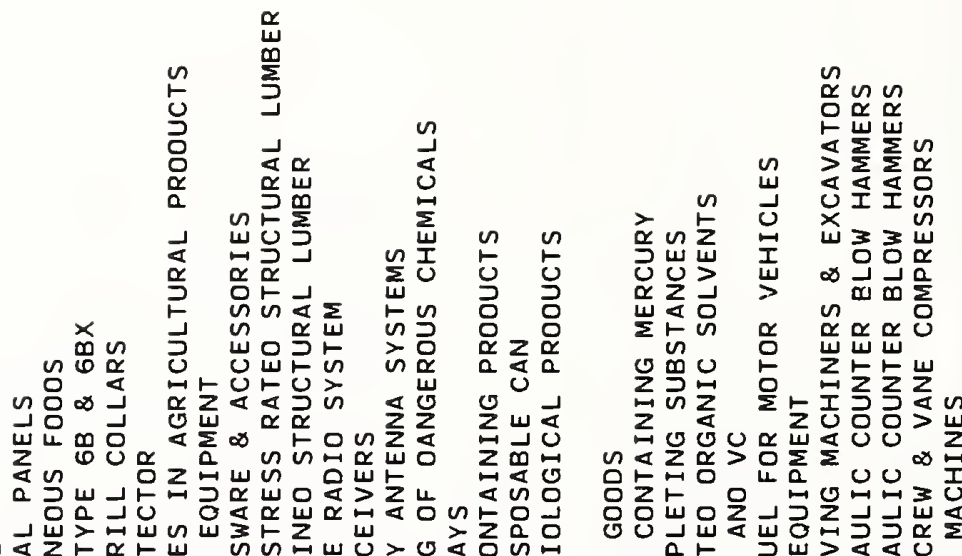

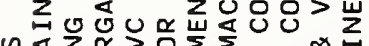

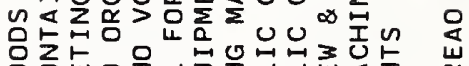

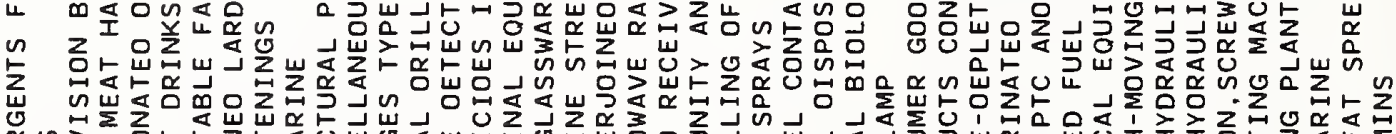

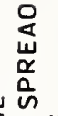

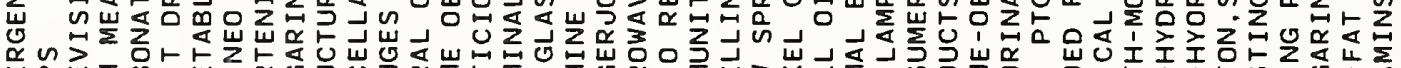

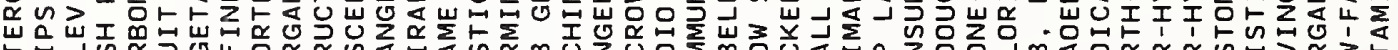

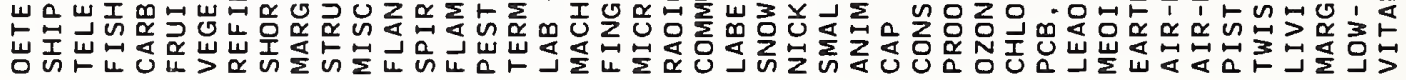

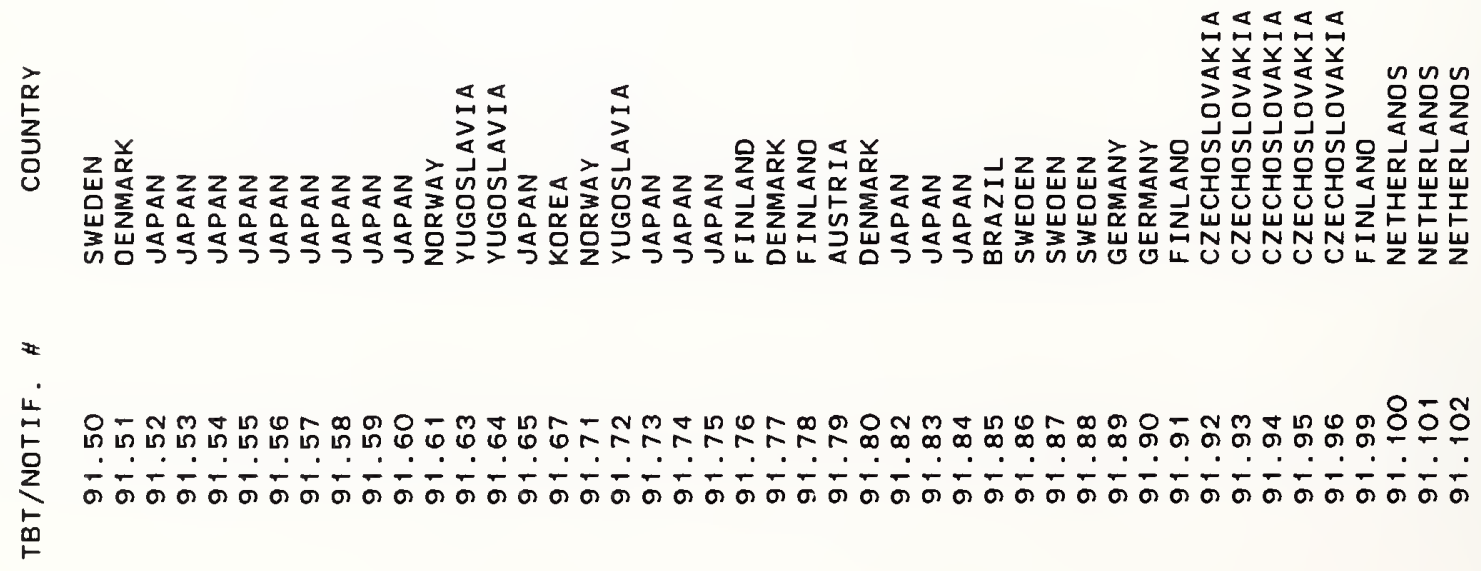




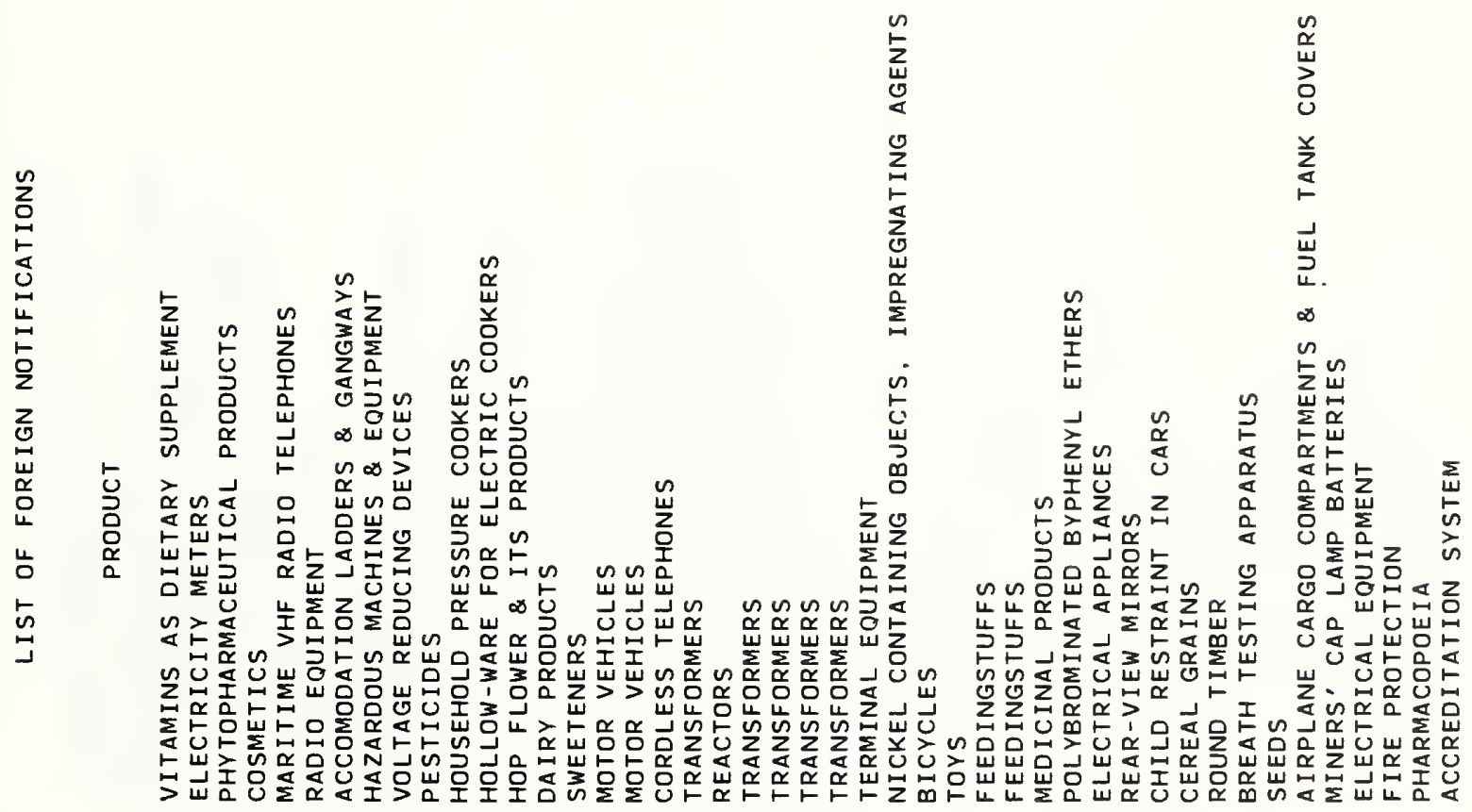

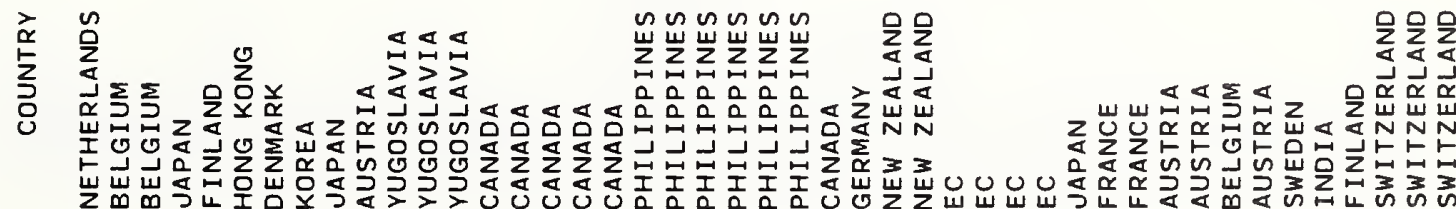

告

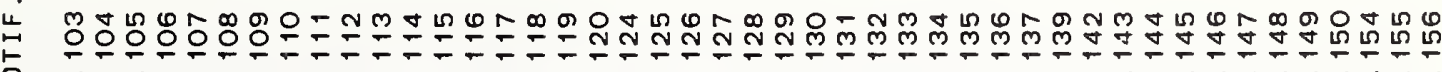

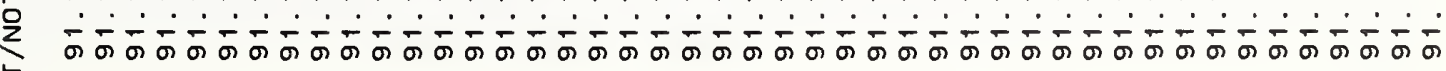

品 


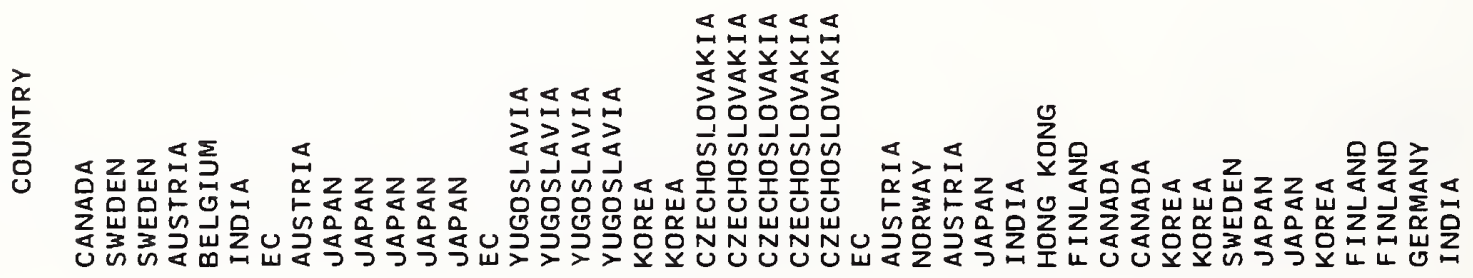

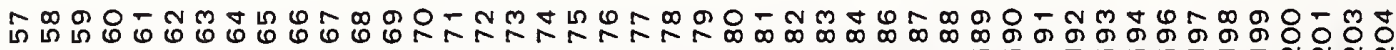

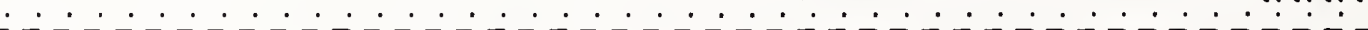

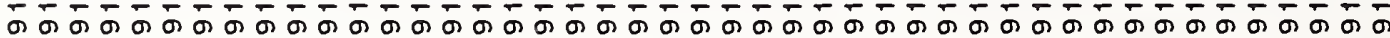



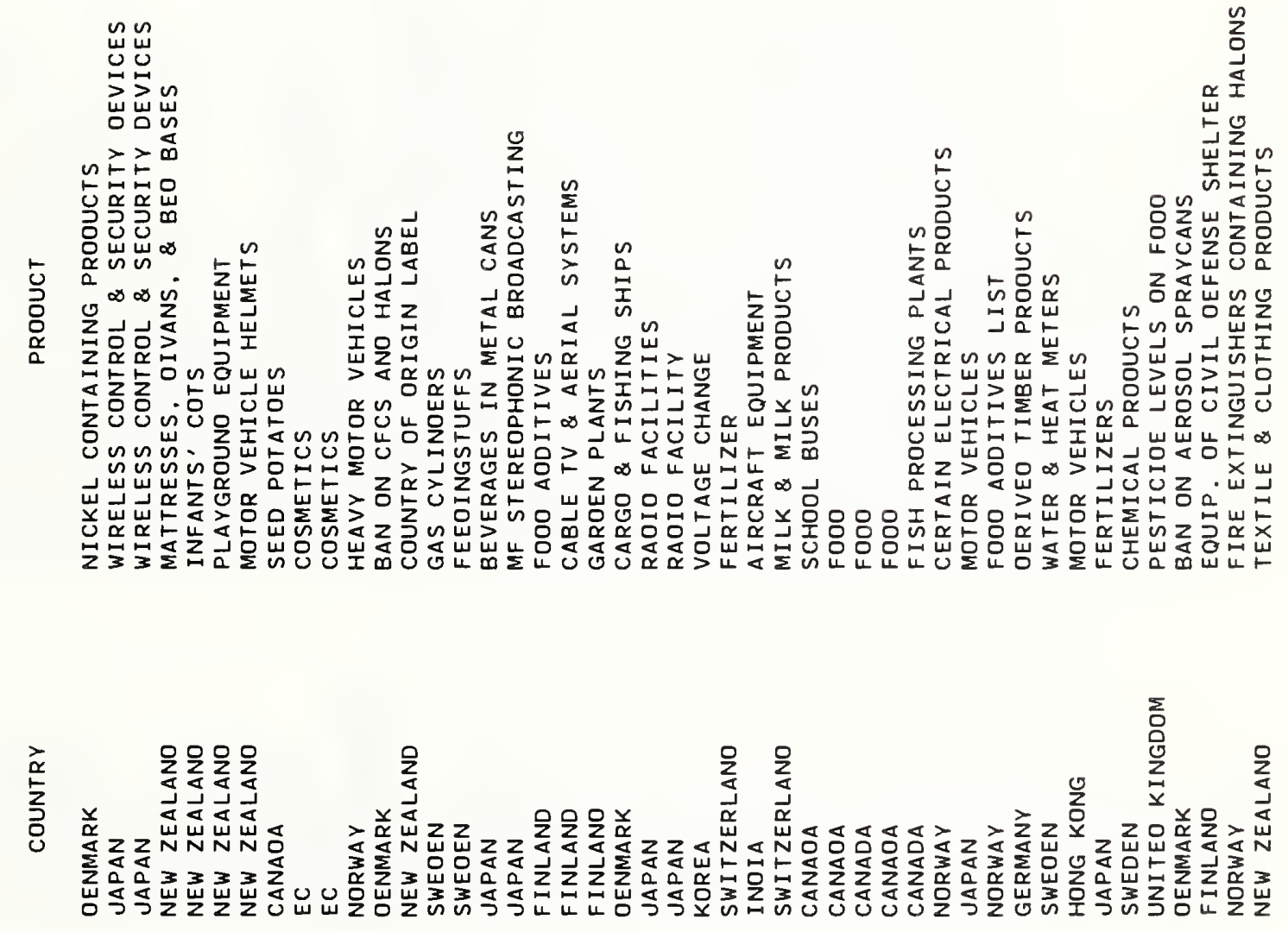

\#

Li

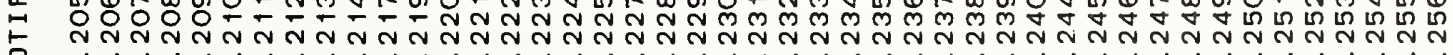

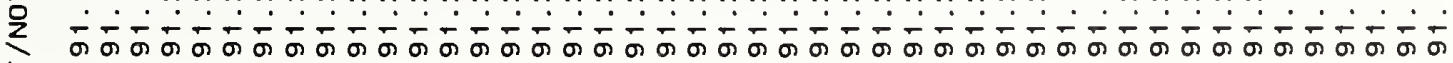
$\stackrel{\mapsto}{\leftarrow}$ 

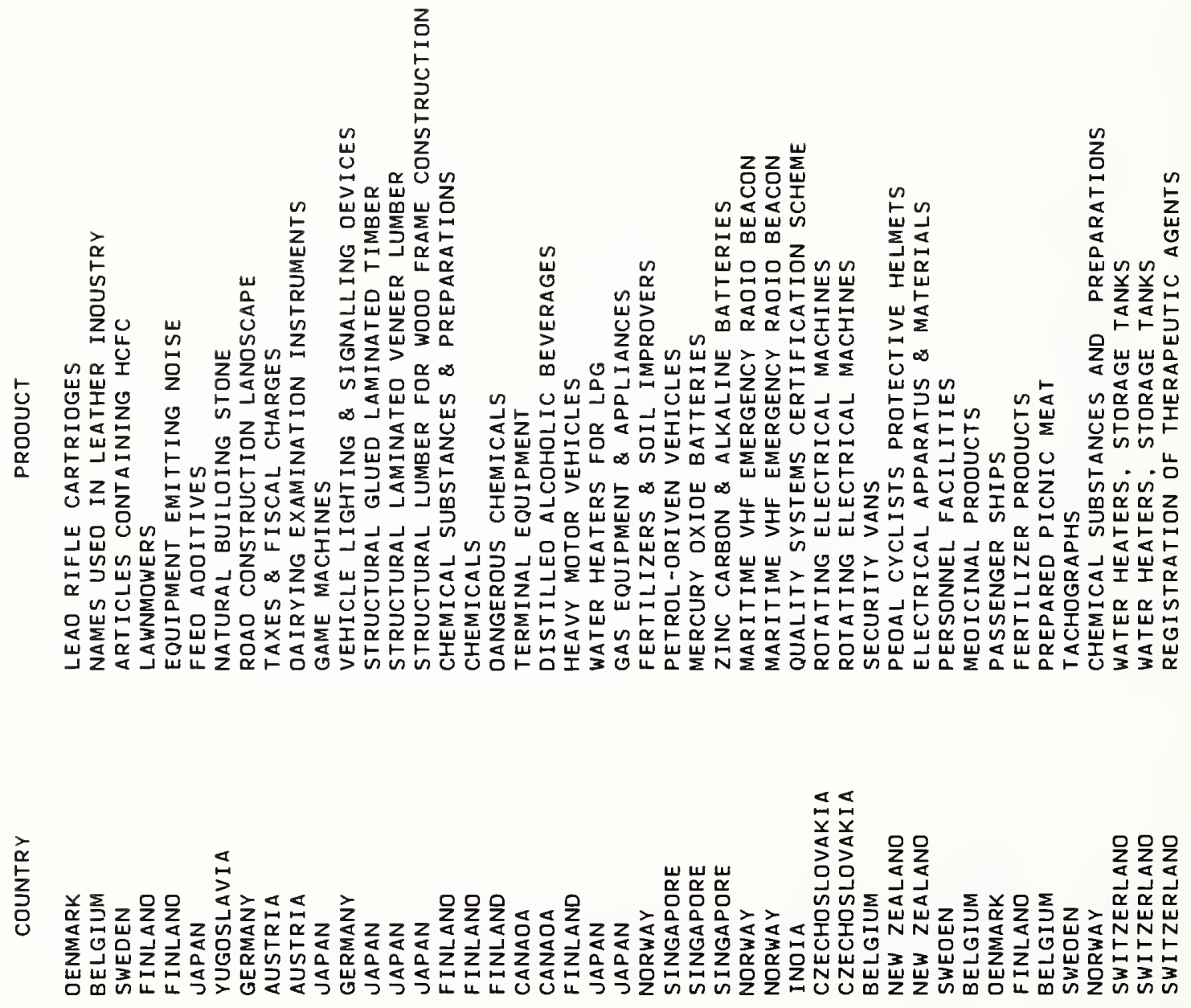
战

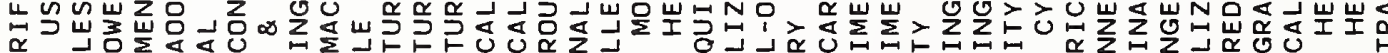

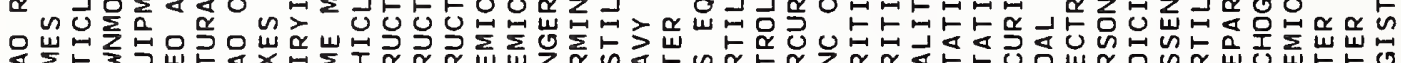

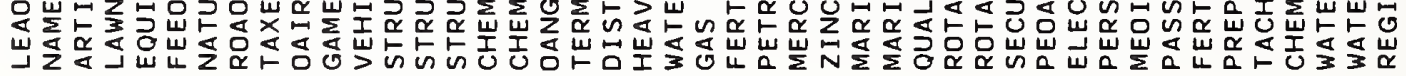

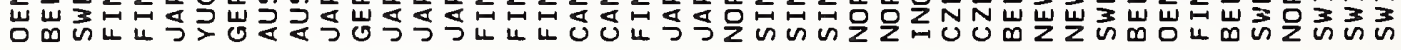

는 点

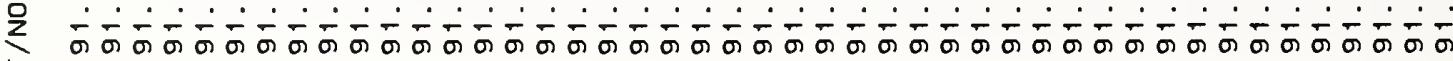



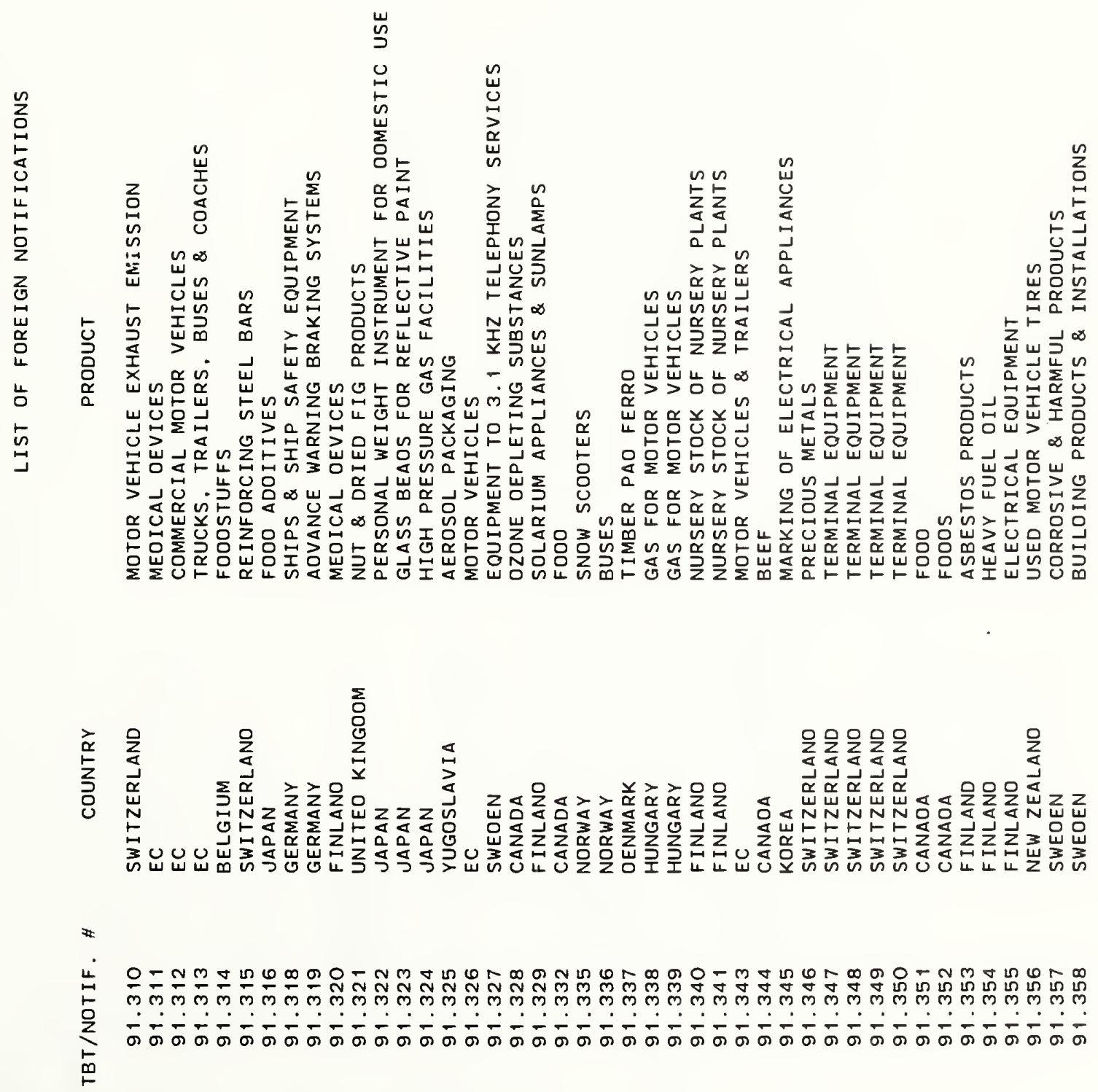

: :

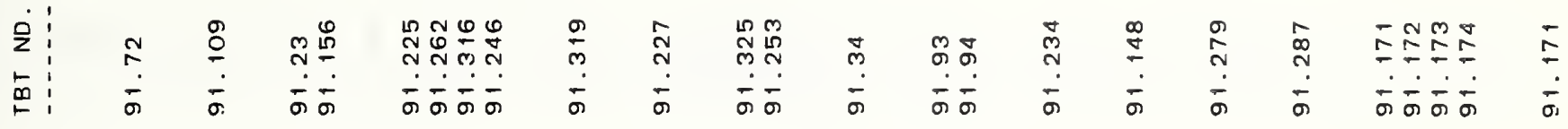

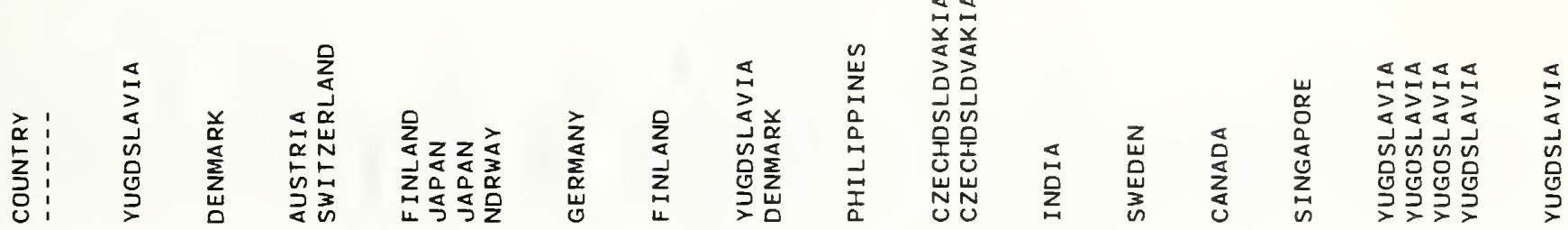

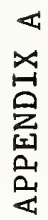

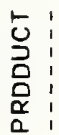

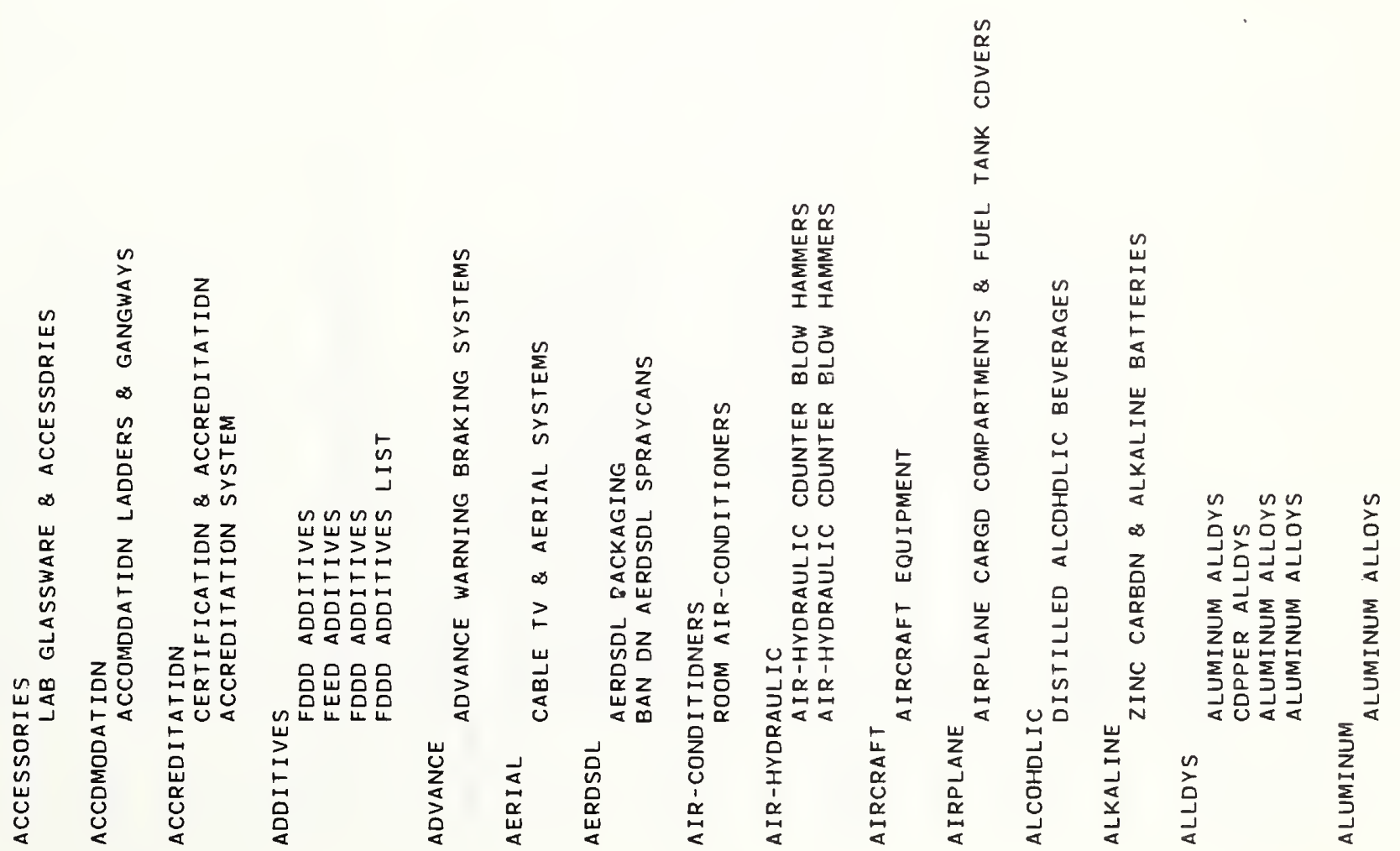



:
กั๊ํำ

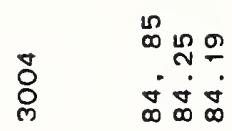

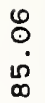

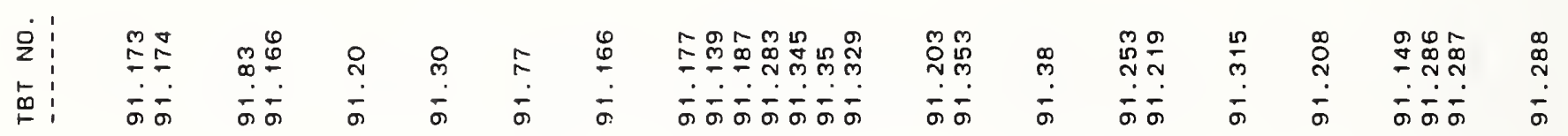

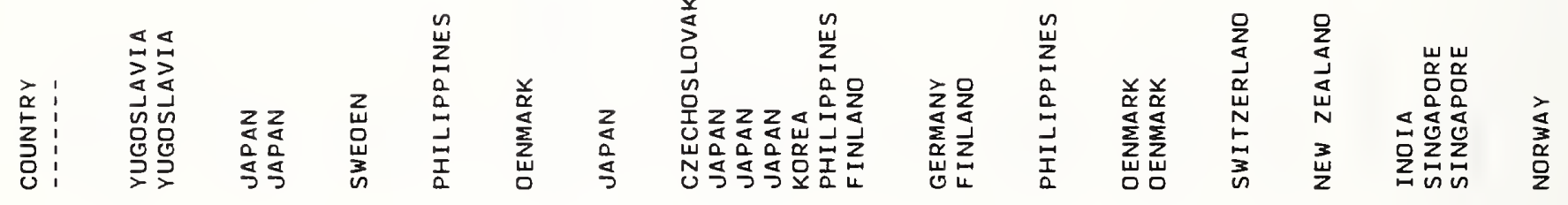

:

प्रा $\frac{1}{2}$

a

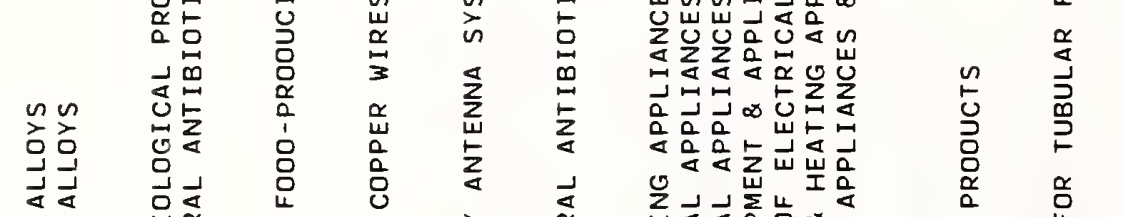


\begin{tabular}{l:l}
0 & \\
\hdashline & 0 \\
0 & 0
\end{tabular}

ర్ల

กั ข

ำ

กั

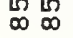

金

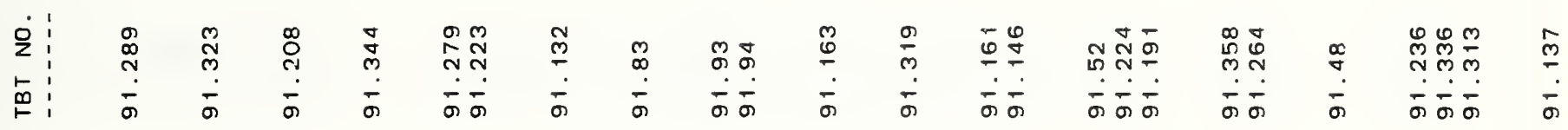

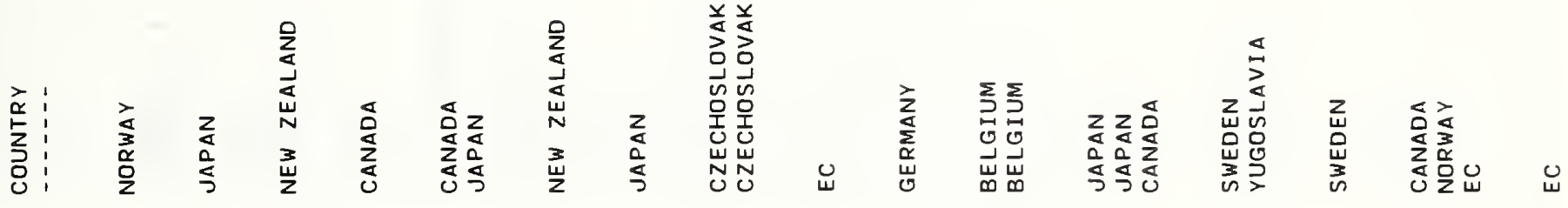

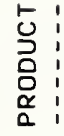

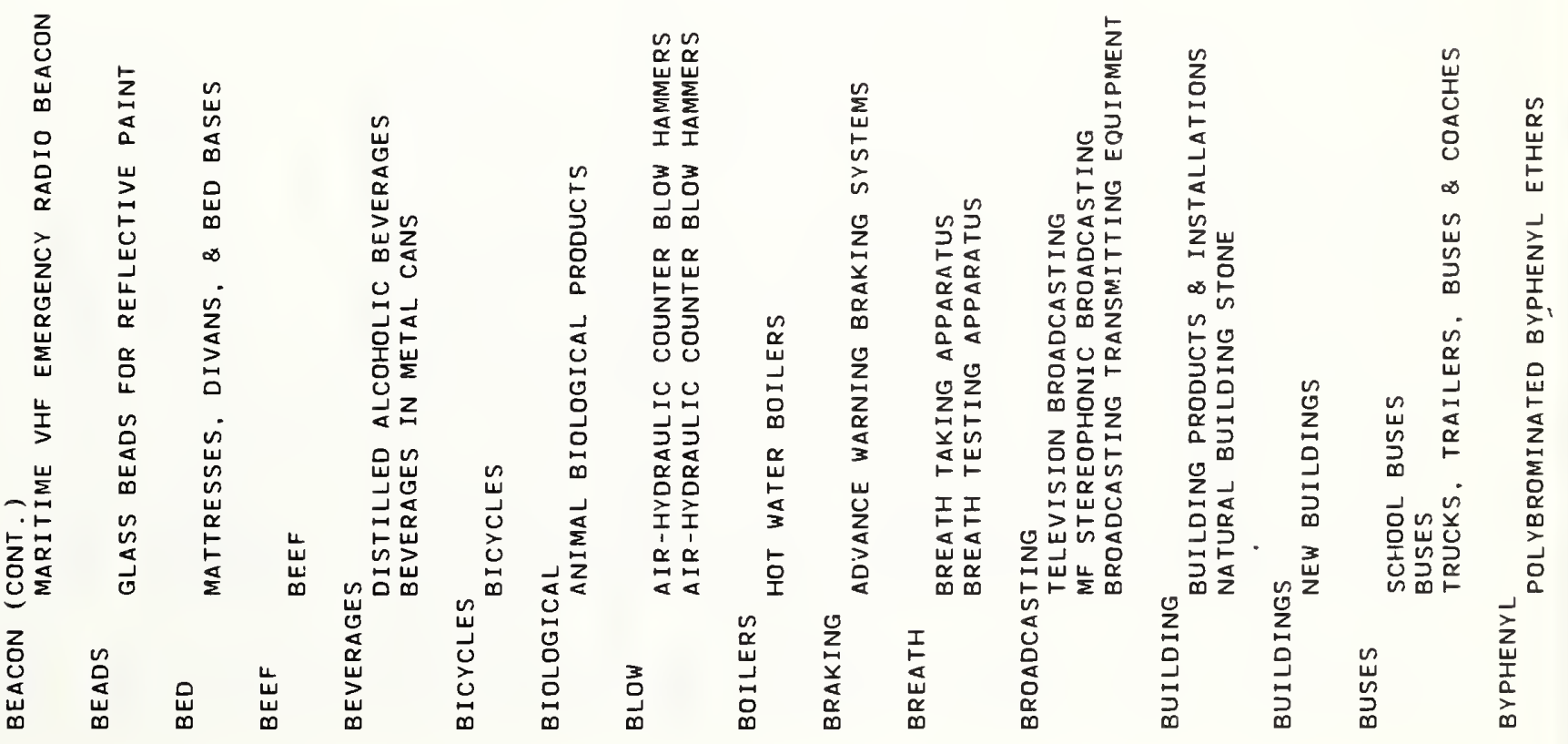




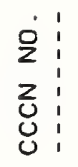

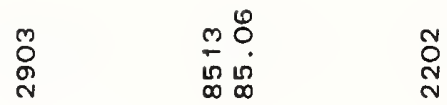

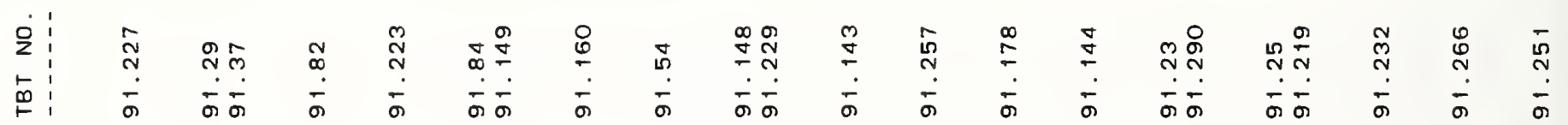

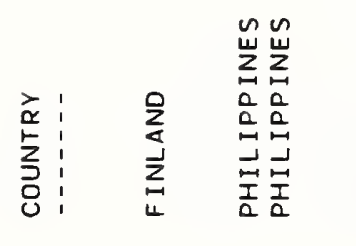

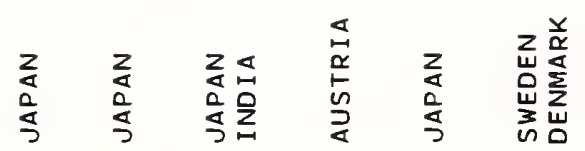

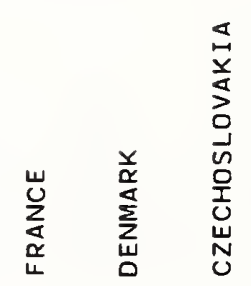

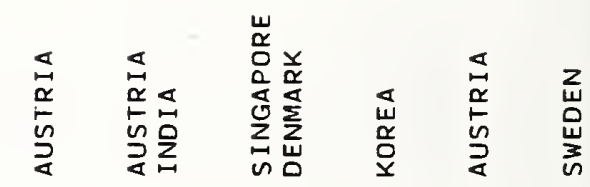

:

足

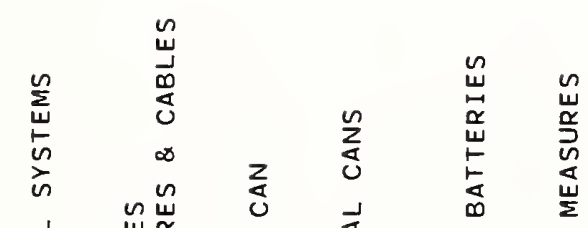

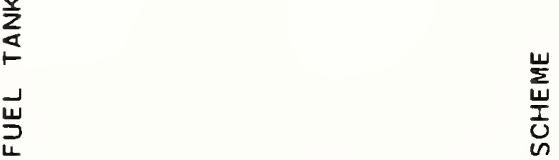

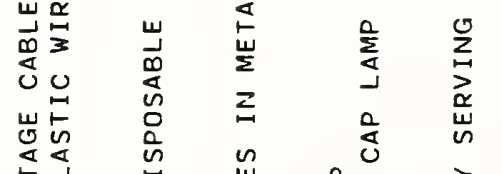

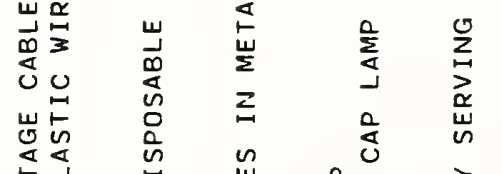

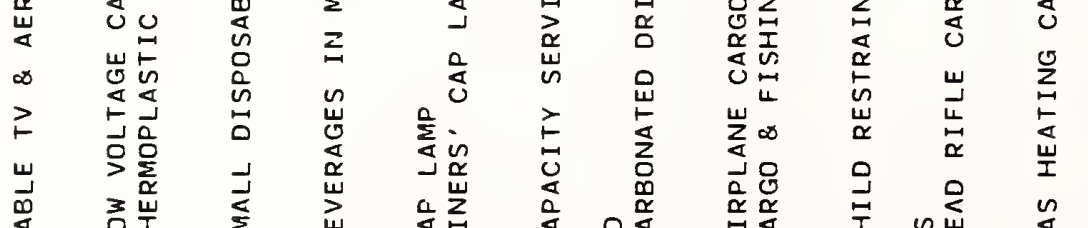

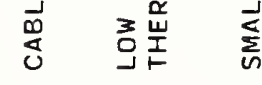

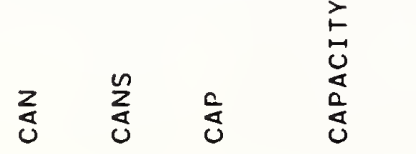

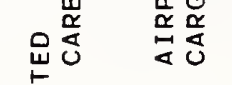
咅

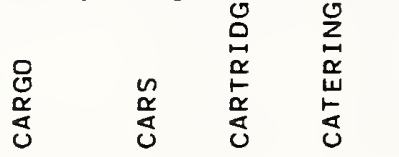
告 峞 


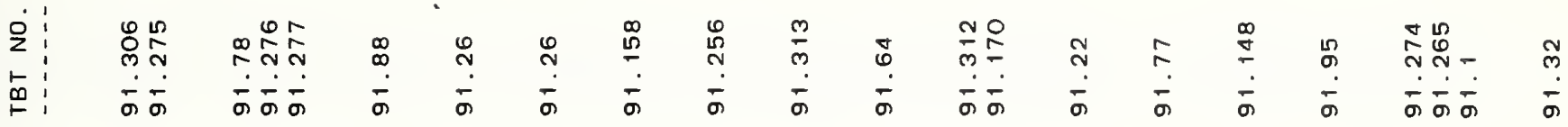

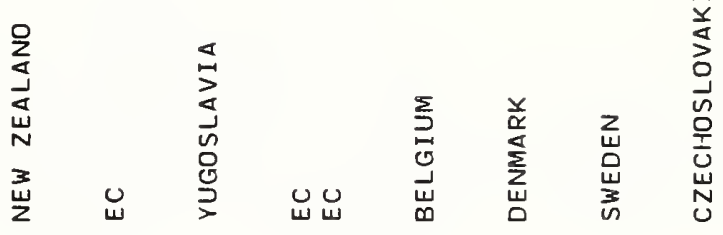

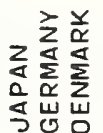

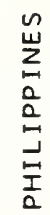

¿্ৰ

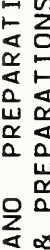

岂出

$\sum_{\substack{u \\ 4}}$

占告

$\stackrel{9}{\supset}$

. $\frac{1}{4} \frac{1}{4}$

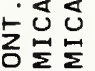

娞殅

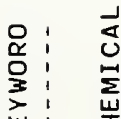

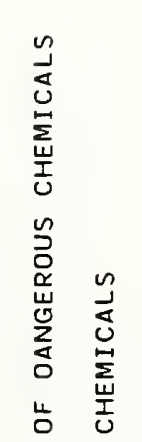

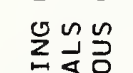

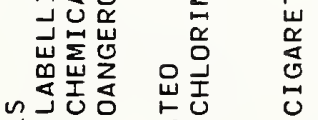

要

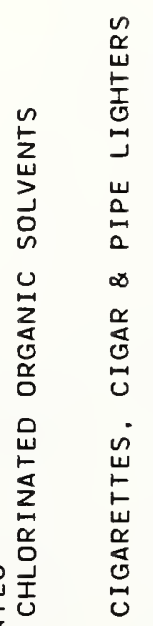

点

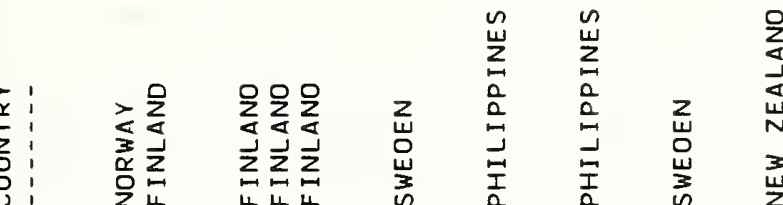

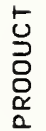

兄

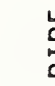

$\begin{array}{l:ll}2 & 0 & 0 \\ 0 & 0 & \infty\end{array}$
岸岁

$\stackrel{\substack{\alpha \\ \stackrel{\sim}{\sim}}}{0}$

产

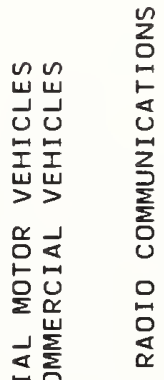

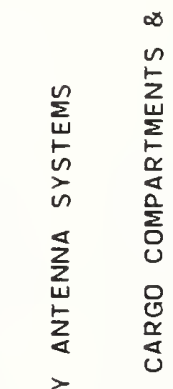

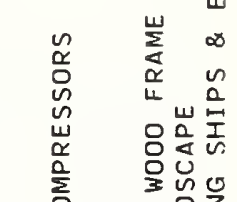

讨

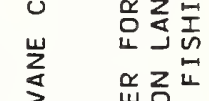

운

品品

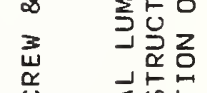

范怘

인맘

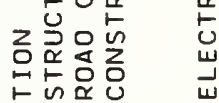

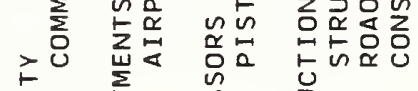

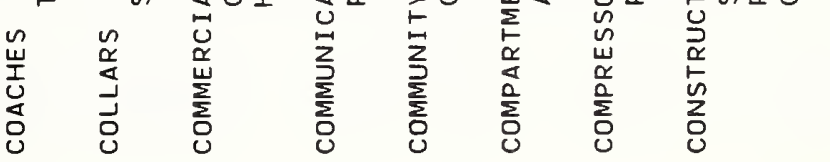

웄

岂

㟧 
ì

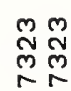

앴욤요

$\frac{1}{\stackrel{2}{2}}$

$\stackrel{n}{\infty}$

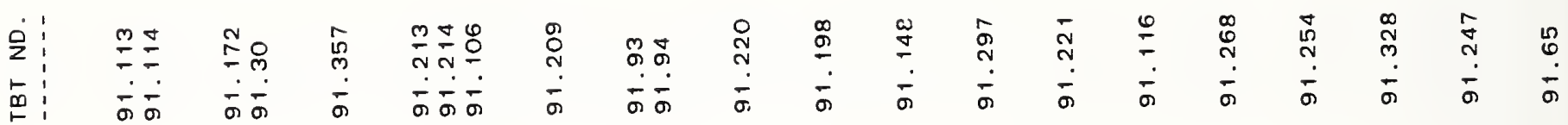

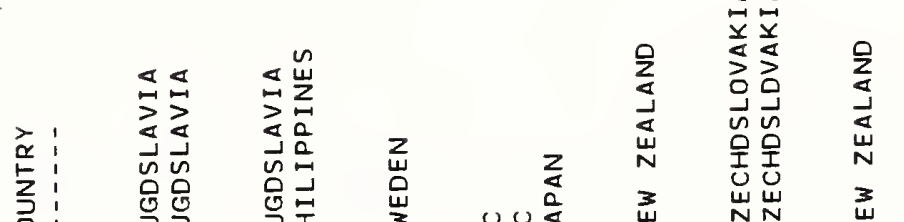

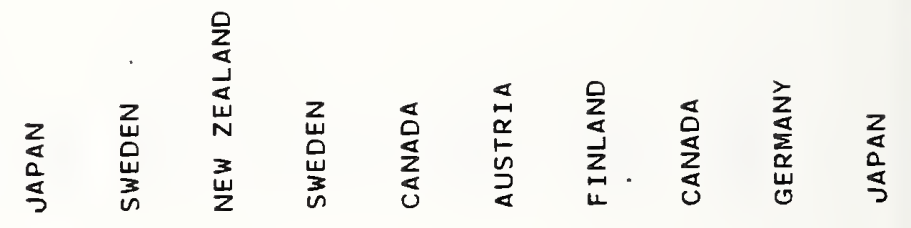

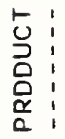

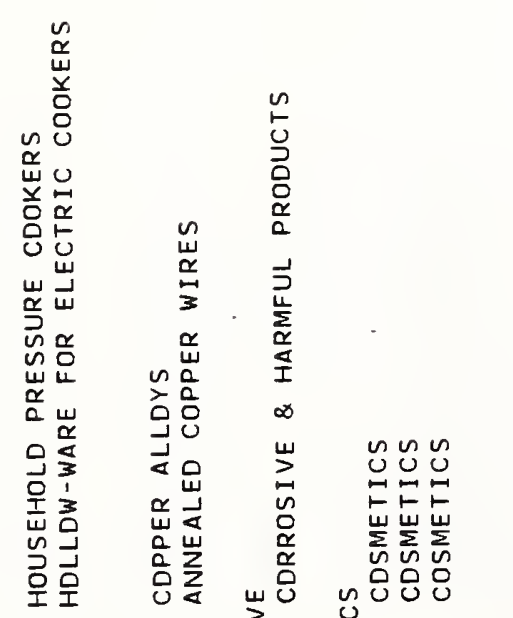

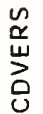

差

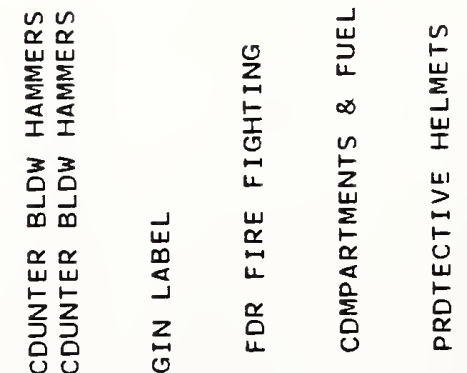

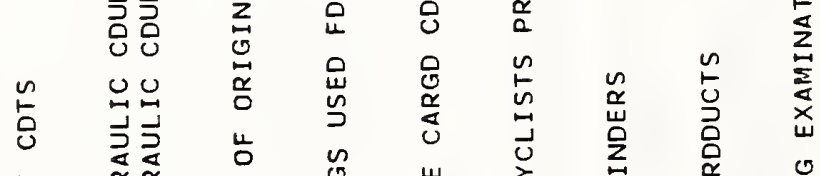

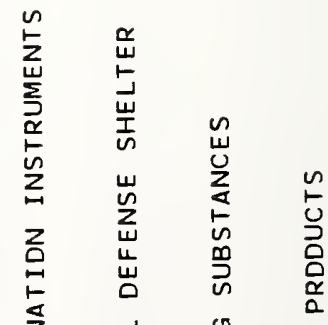

矛总总 


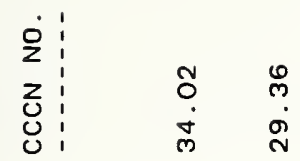

옹

กูํำ 을

$\stackrel{m}{N}$

เูก กู

这

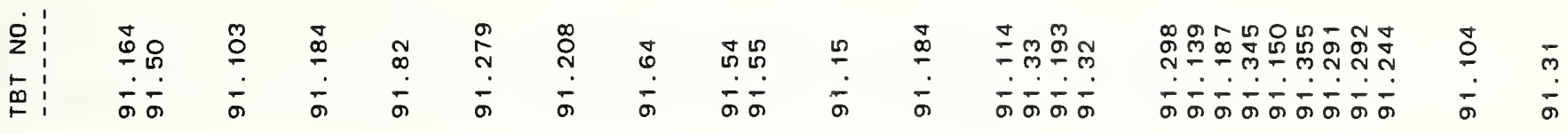

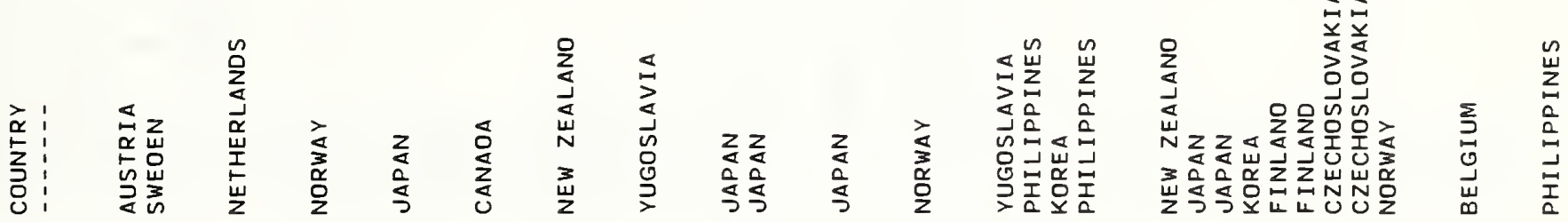

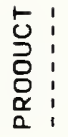

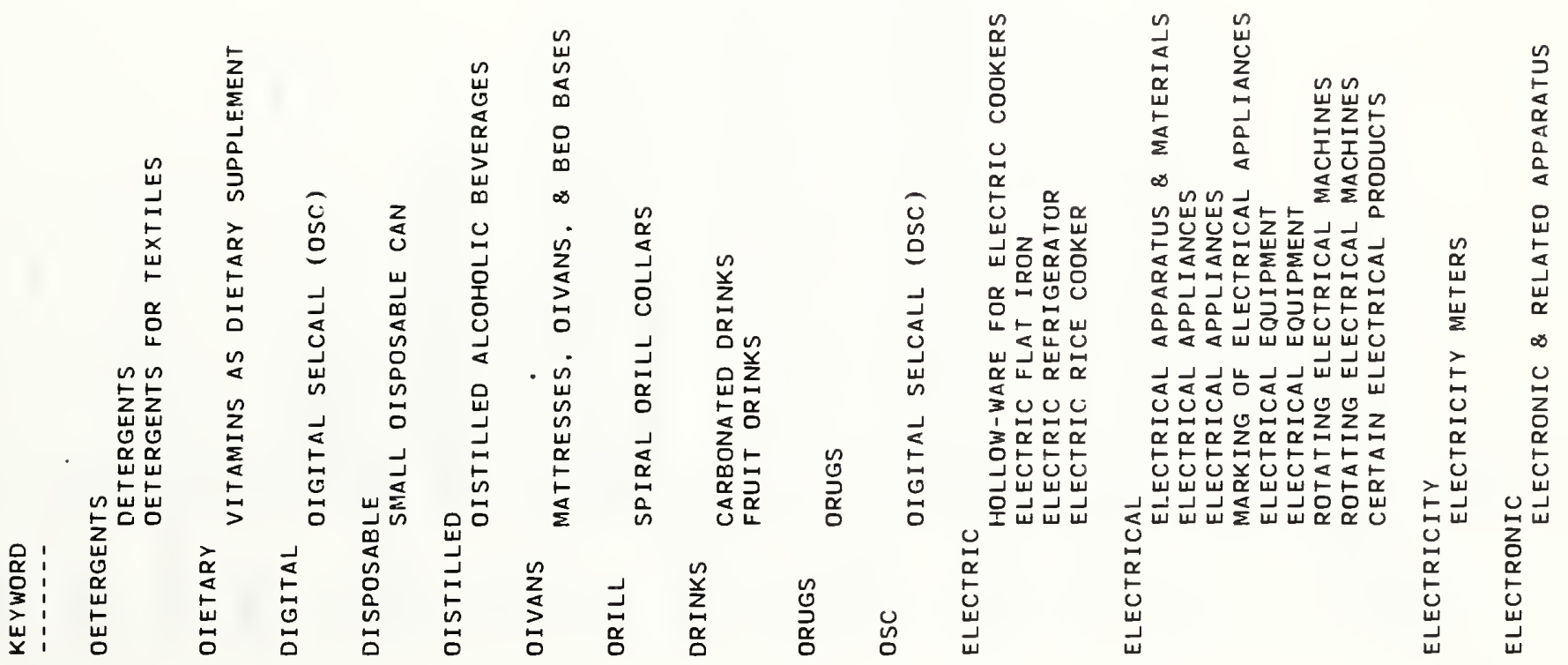




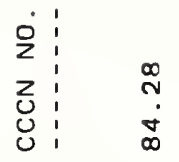

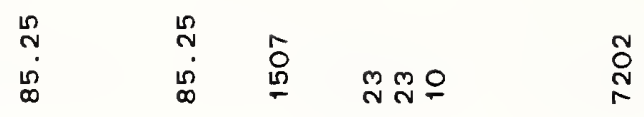

$\bar{m}$

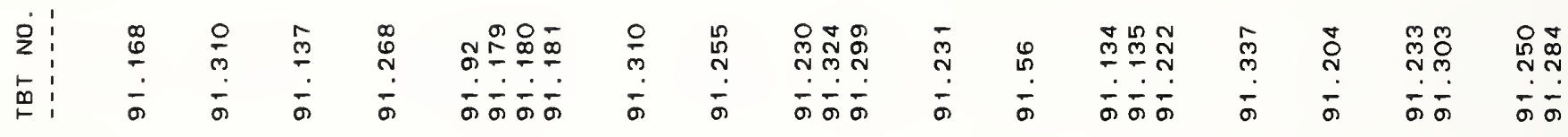

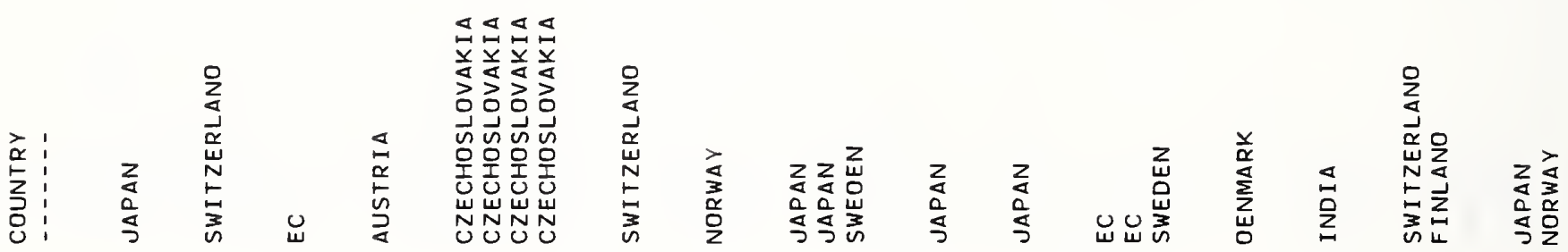

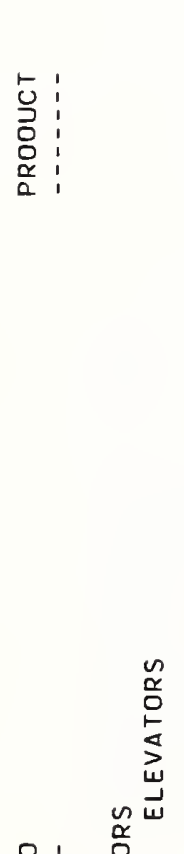

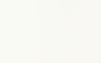

.

(I)
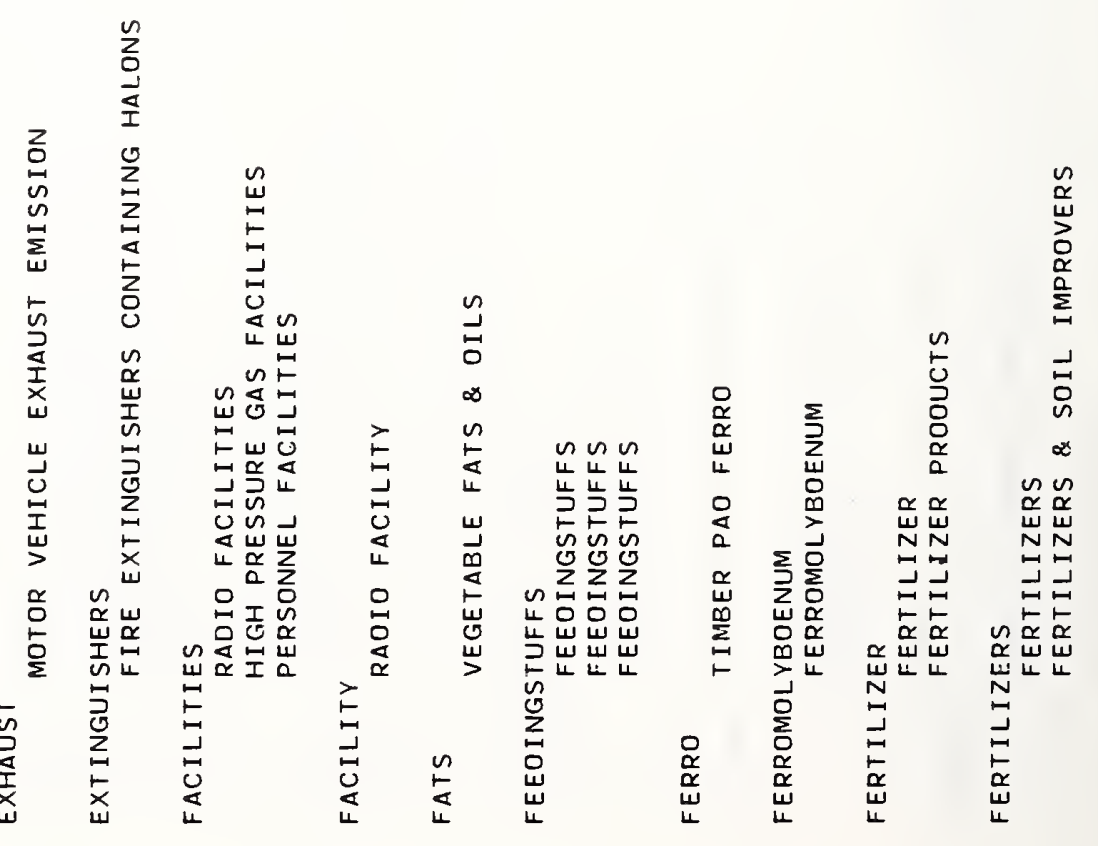
\begin{tabular}{l|l}
2 \\
2 \\
2
\end{tabular}

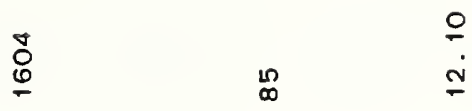

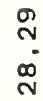

望)

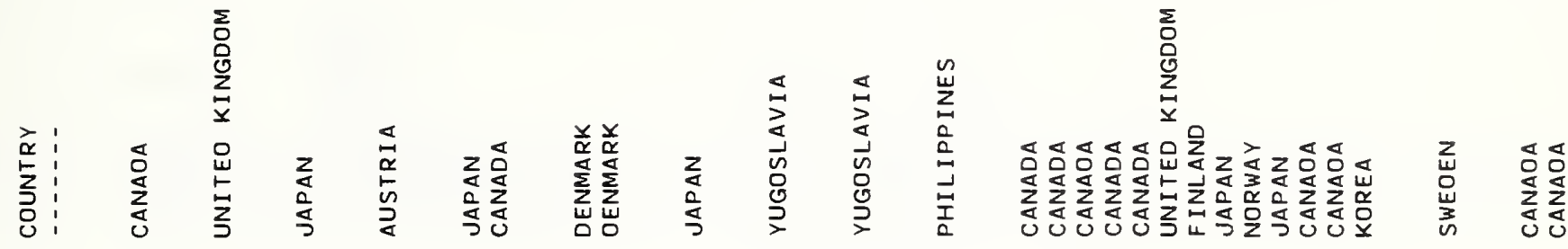

:

$\sum_{1}^{0}$

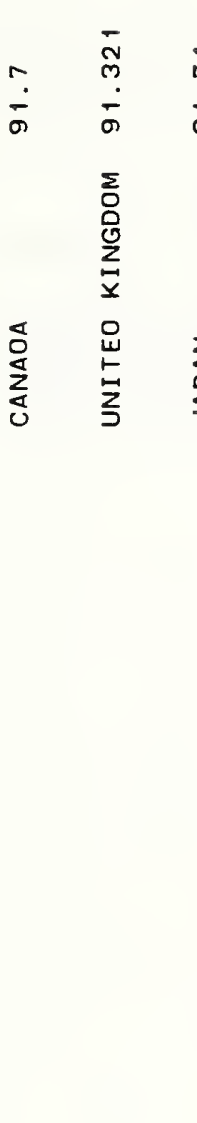

$\infty$
$u$
$a$
$\frac{I}{n}$

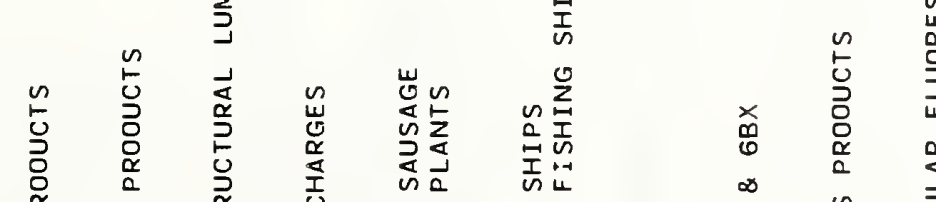

8

2

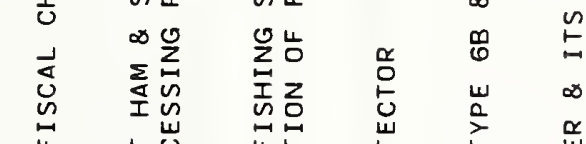

垈点

井

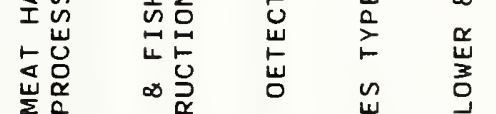

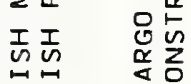

晟

(

แnnu

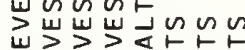

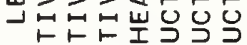

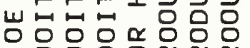

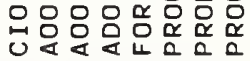

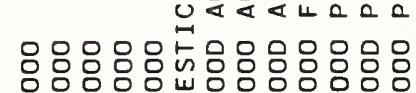

京

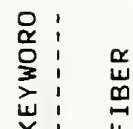

运造

踏

总

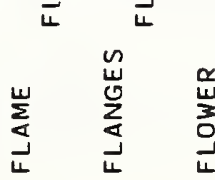

岂

응

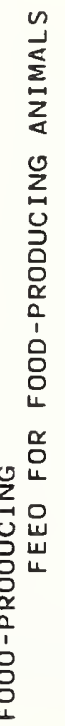

ñ 
i:

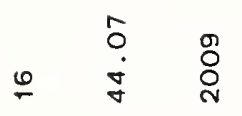

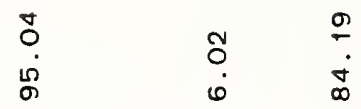

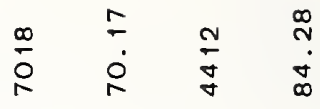

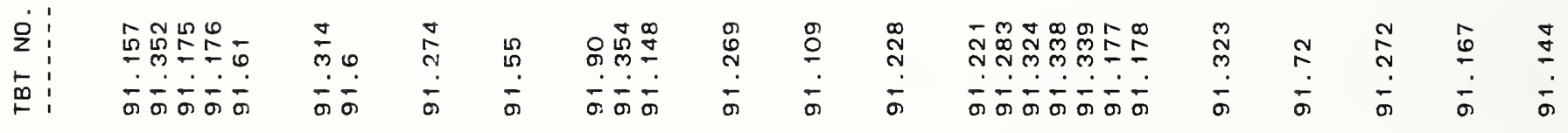

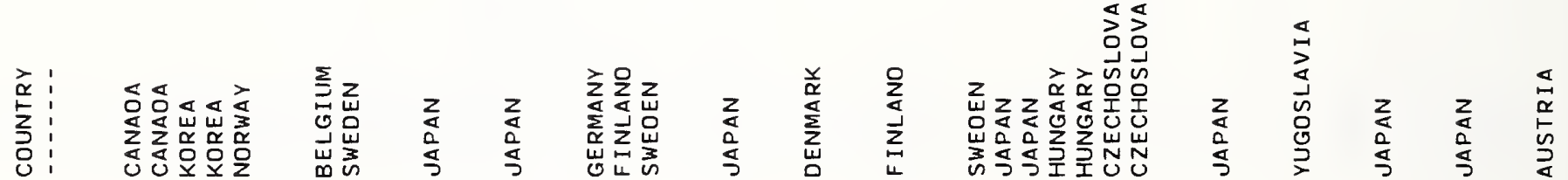

:

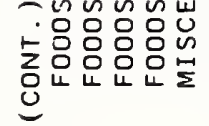

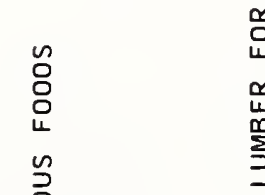

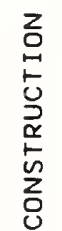

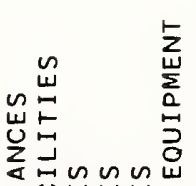

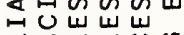

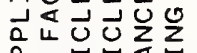

的穴牙近

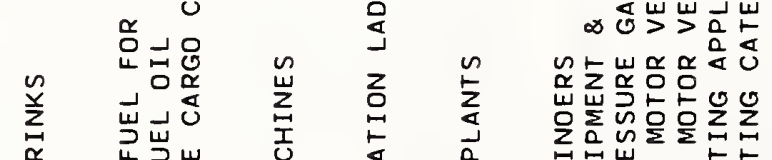

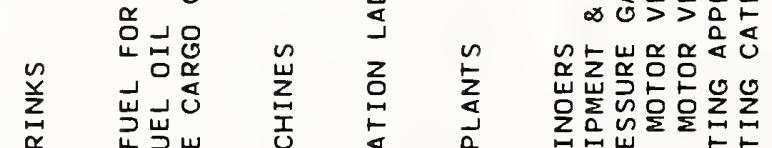

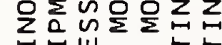

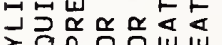

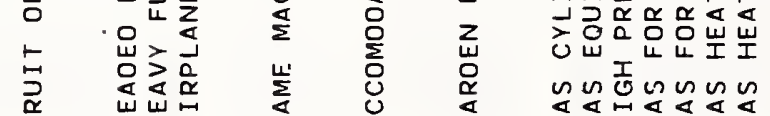

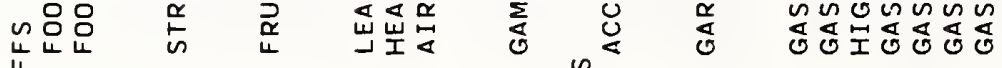

害

嘉言

岁

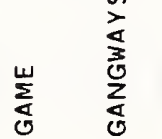

苑

\&

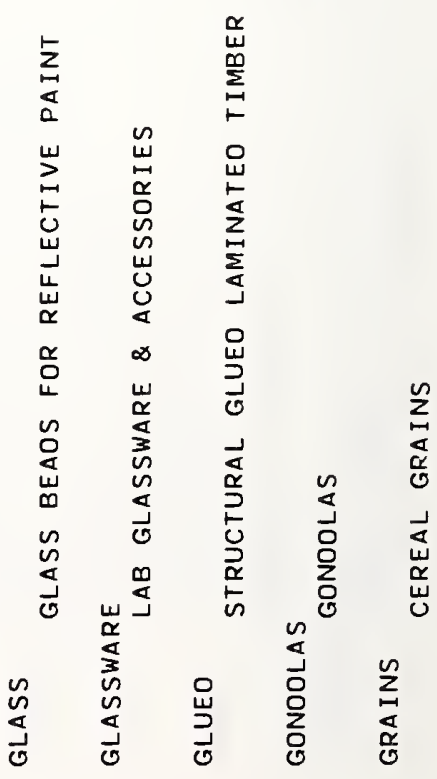




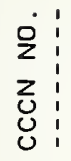

$\stackrel{8}{\circ}$

बे

$\stackrel{\varphi}{\grave{\infty}}$

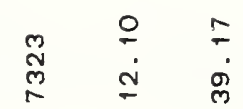

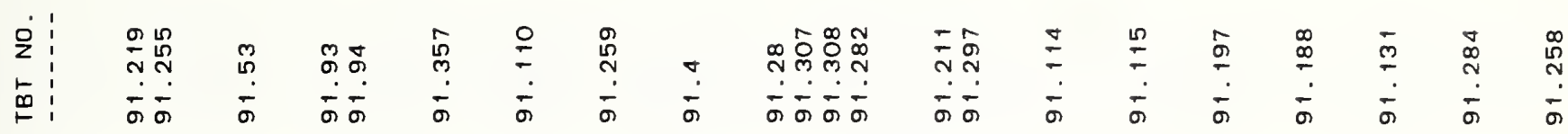

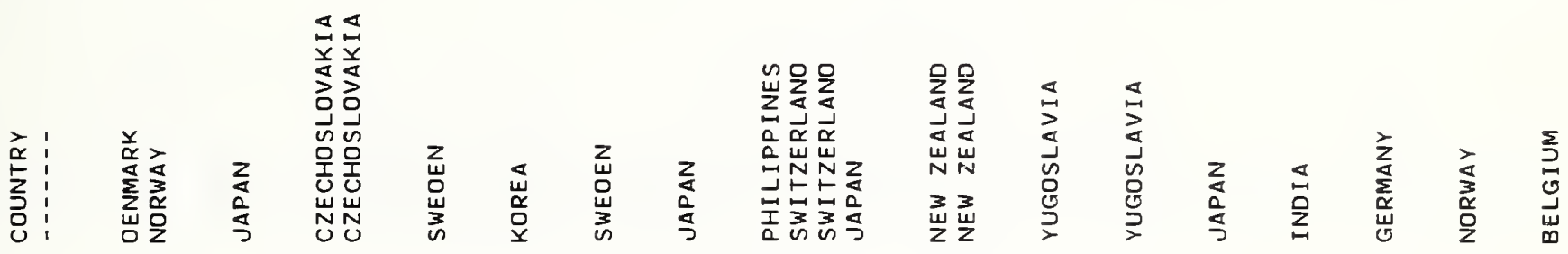

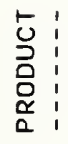

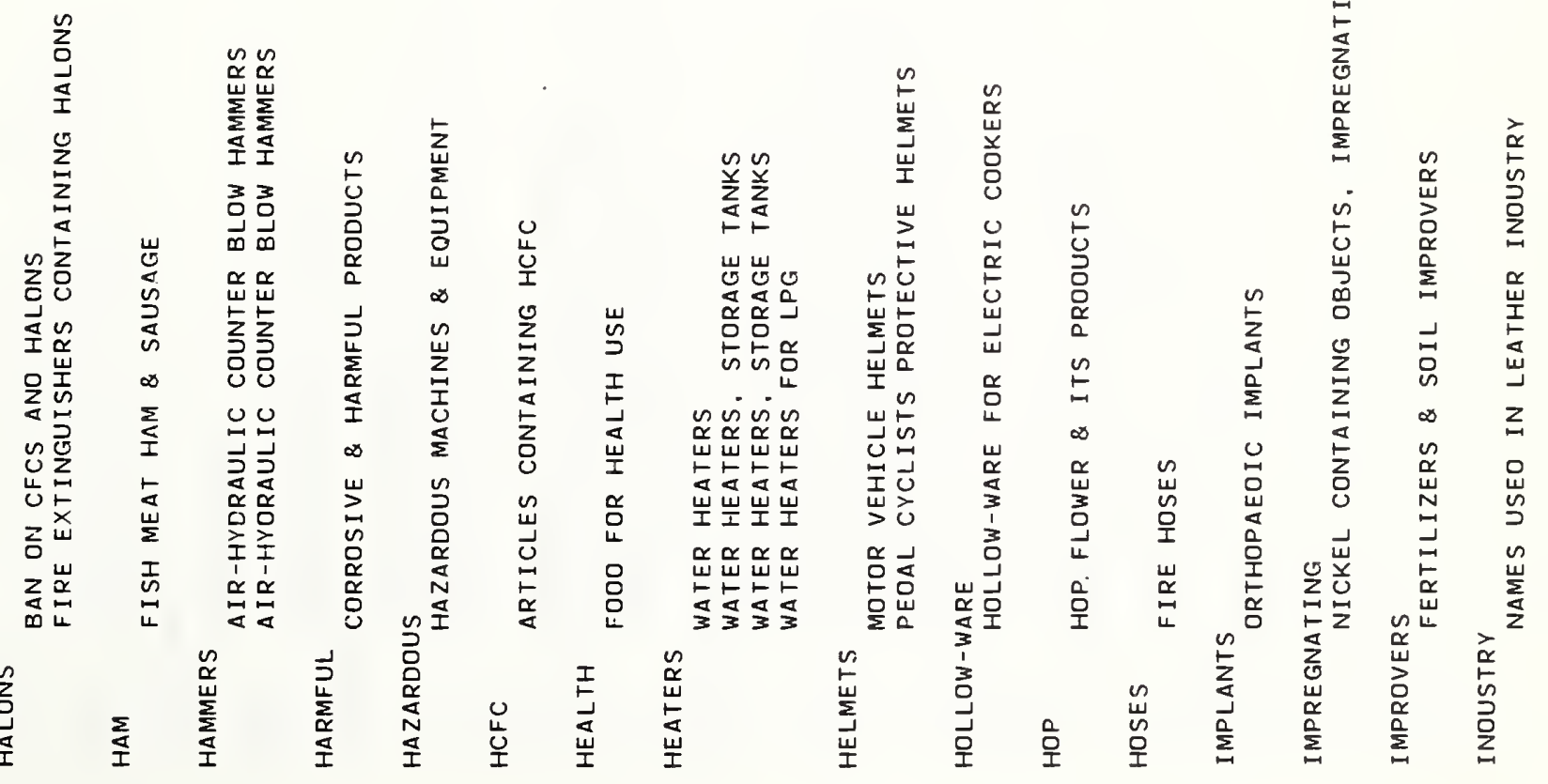




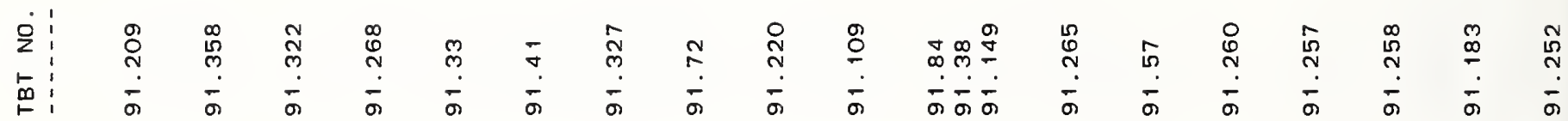

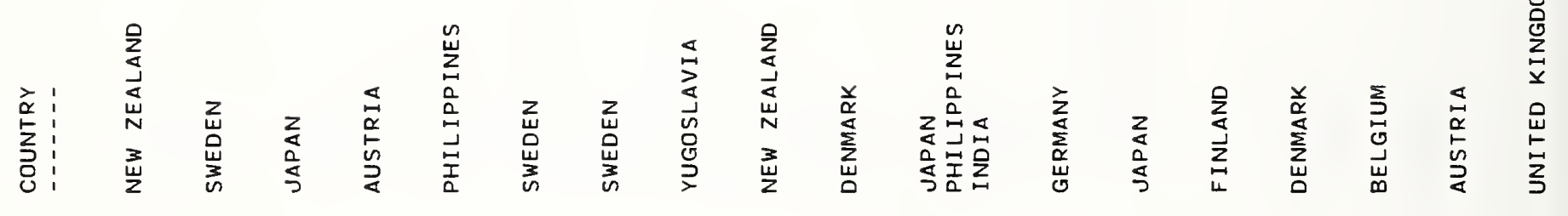

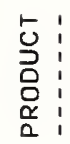

㟧

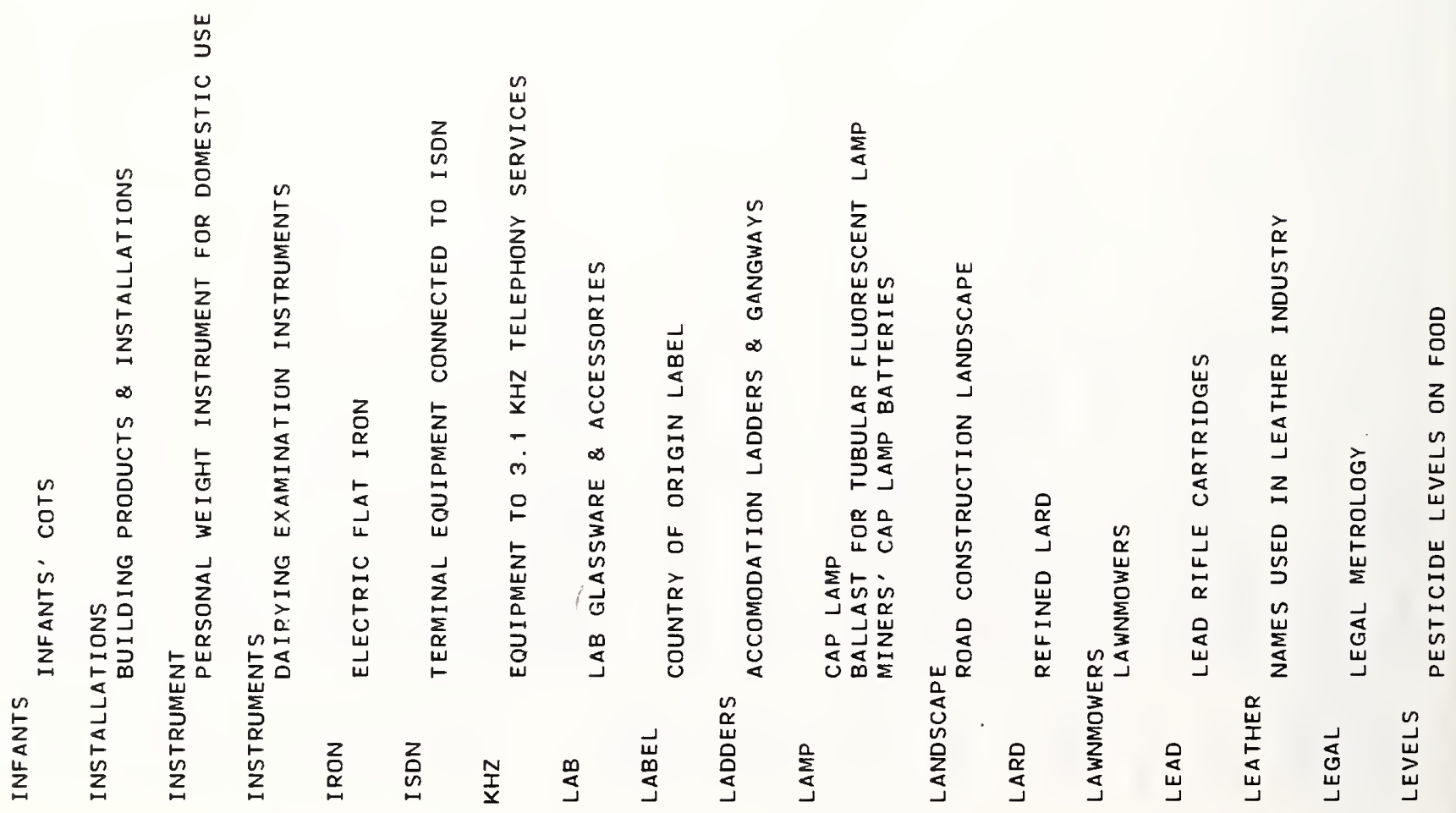




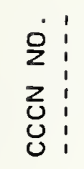

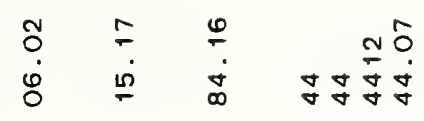

娄

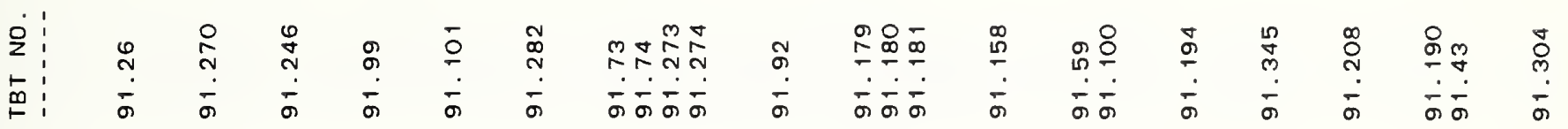

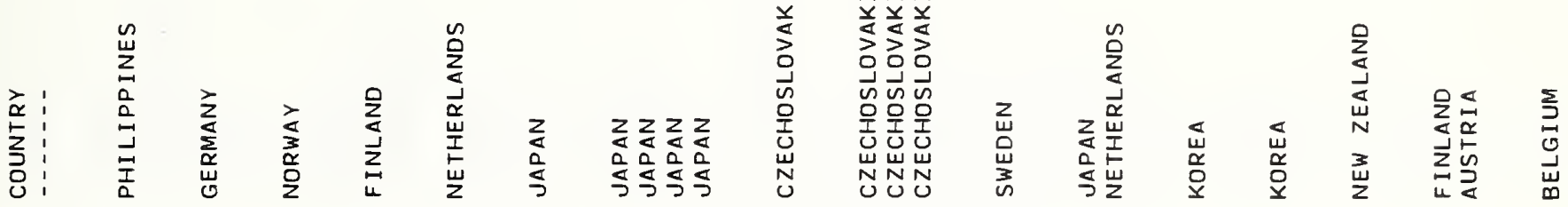

产

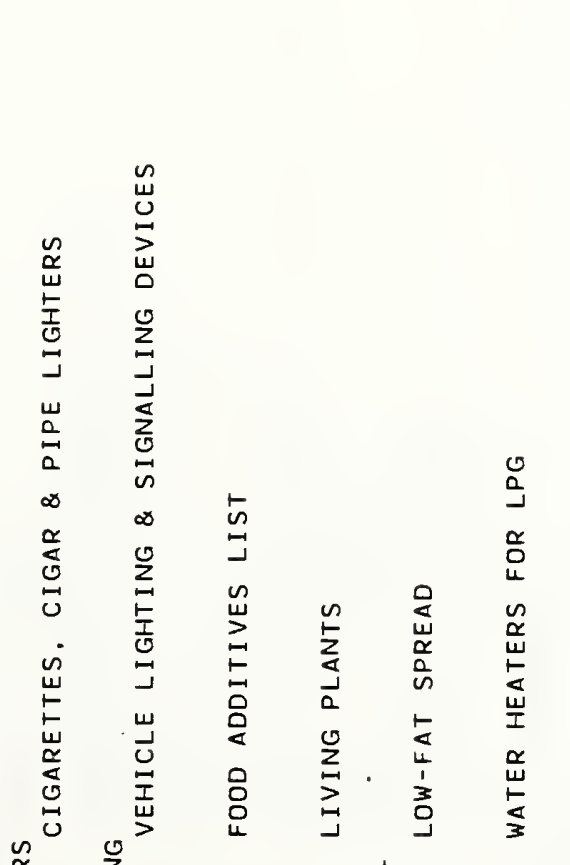<smiles>[CH]1CCC2CCC12</smiles><smiles>C1CCCCC1</smiles>

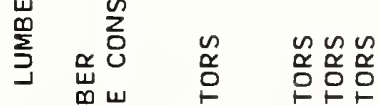

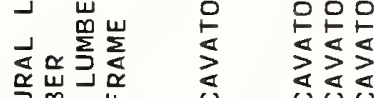

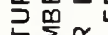

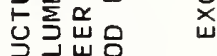

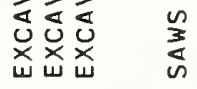
足地

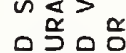

$\infty$

$\infty \infty \infty \quad \frac{\alpha}{4}$

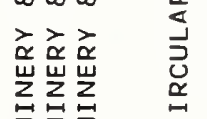

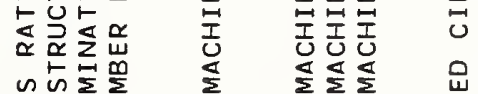

紋主品

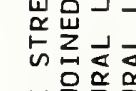

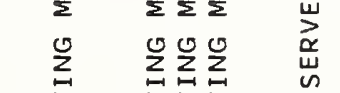

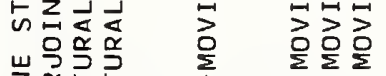

咅

峏岕记足足

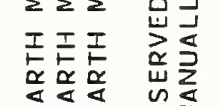

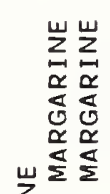

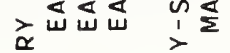

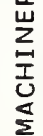

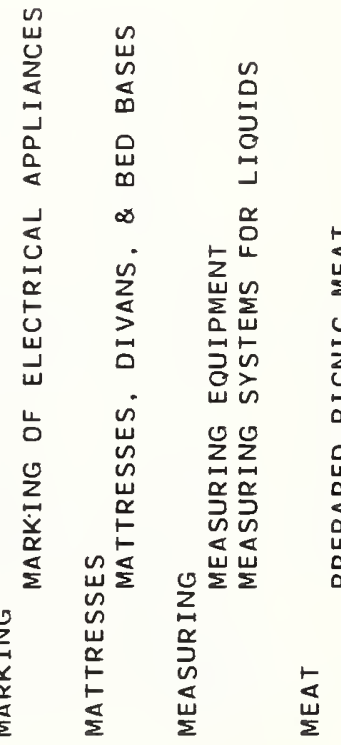


$\begin{array}{l:lll}2 & & & \\ z & & 5 & \\ 0 & 0 & 0 & 0\end{array}$

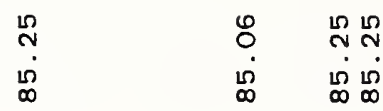

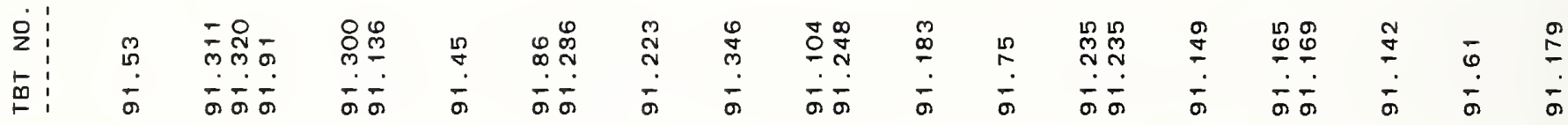

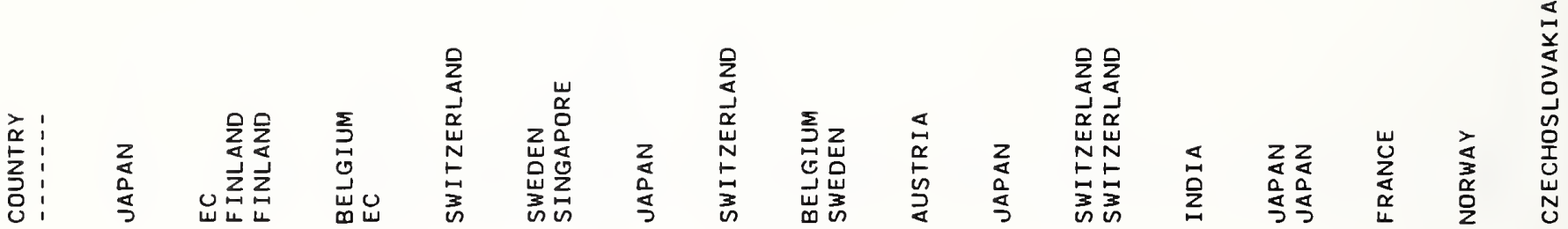

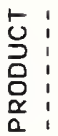

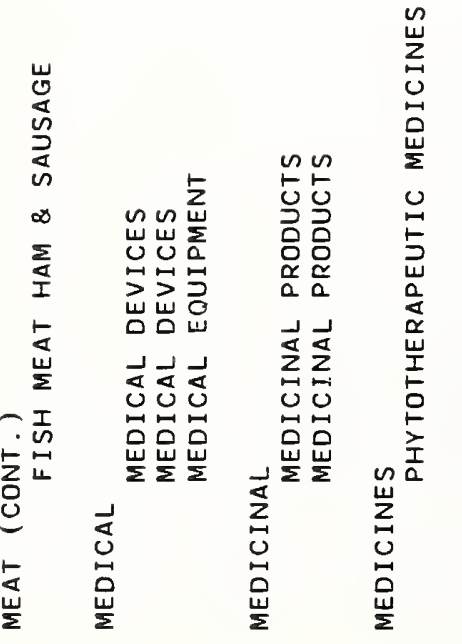

总

空兽

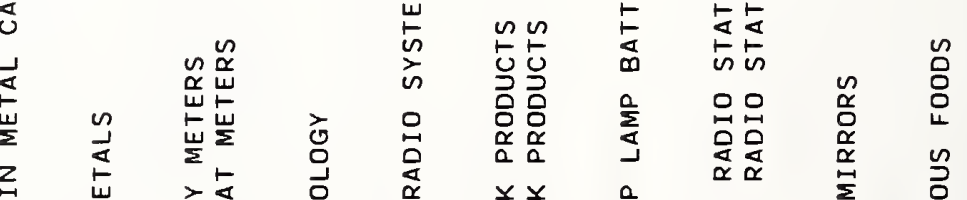

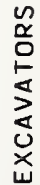

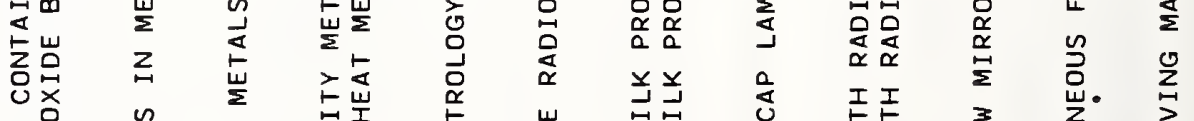




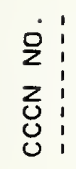

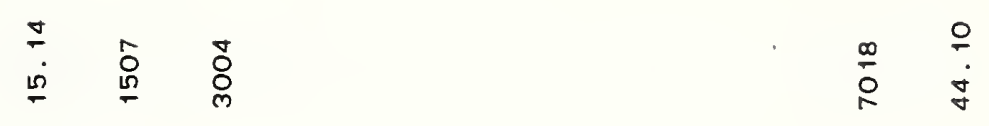

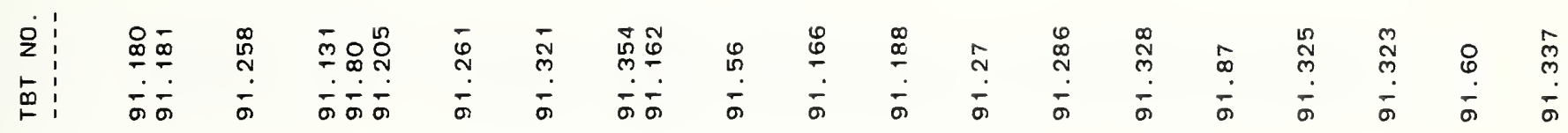

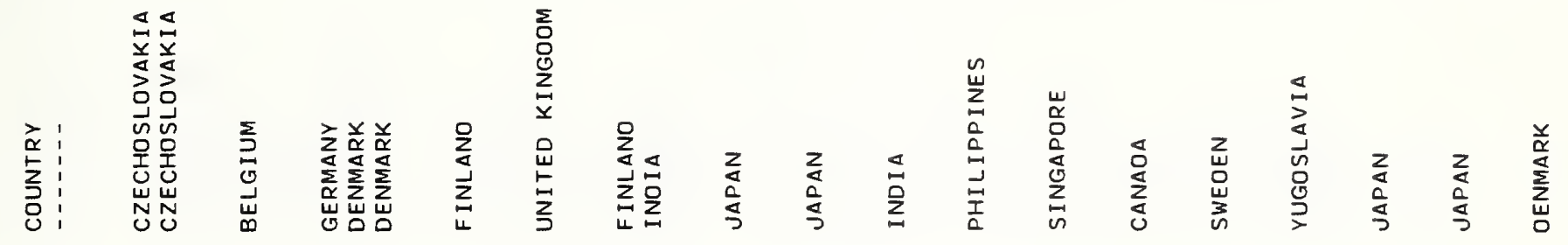

䓂:

营

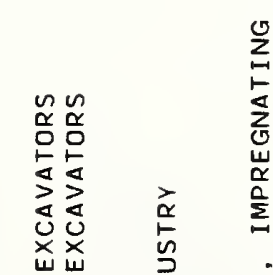

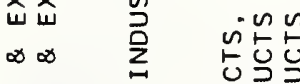

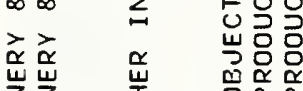

点

西

$000 \%$ 


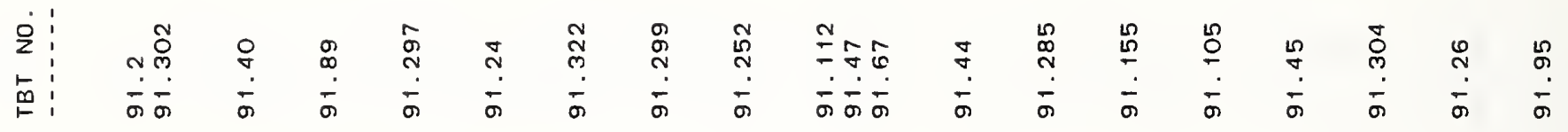

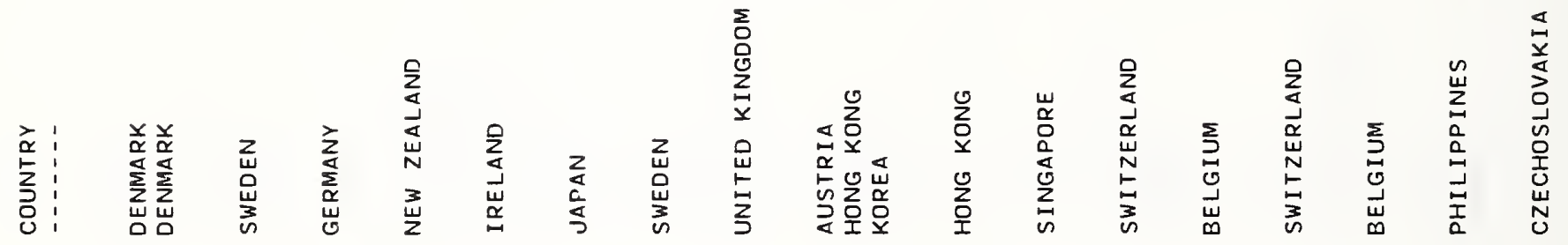

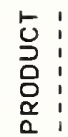
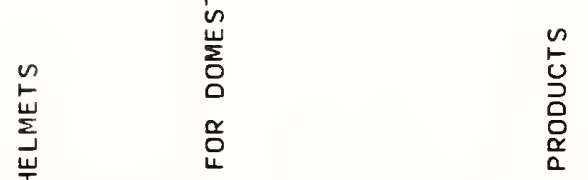

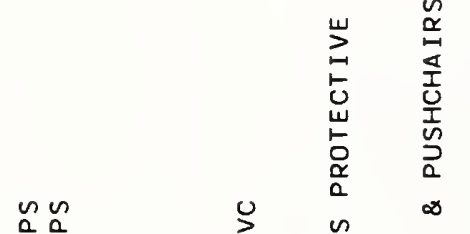

운

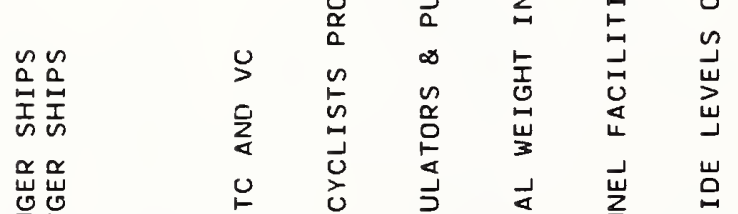

岕岕

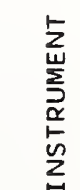

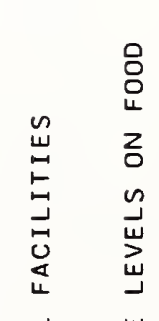

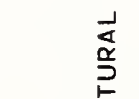

幽出

u

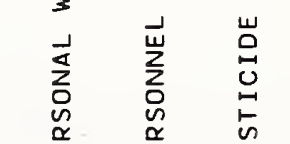

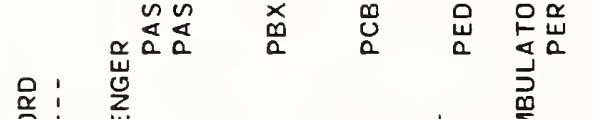

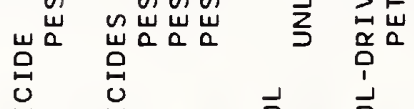

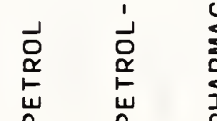

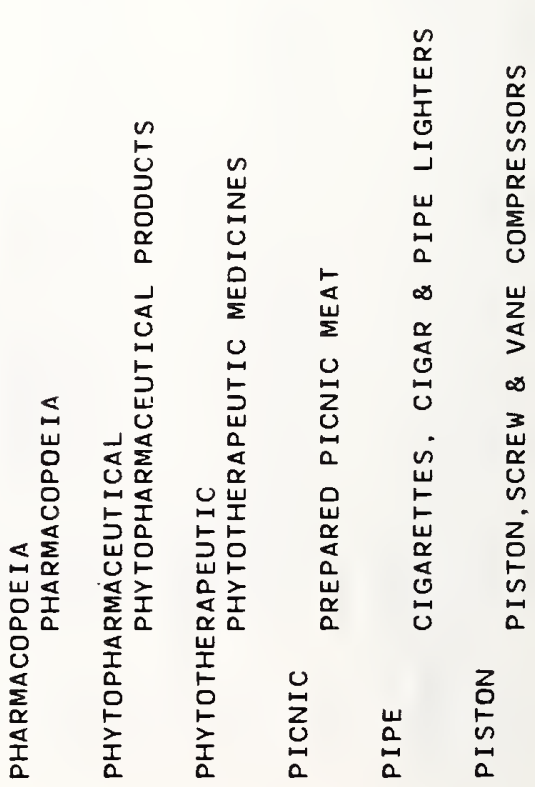




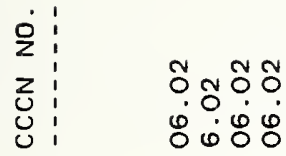

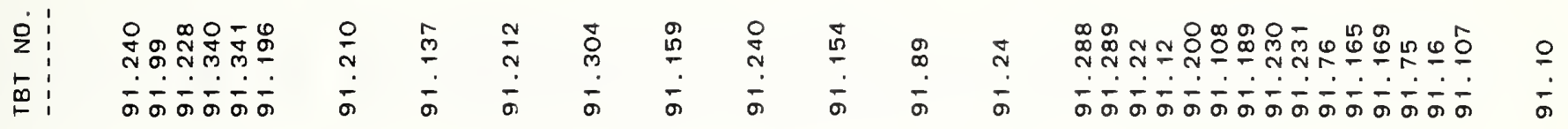

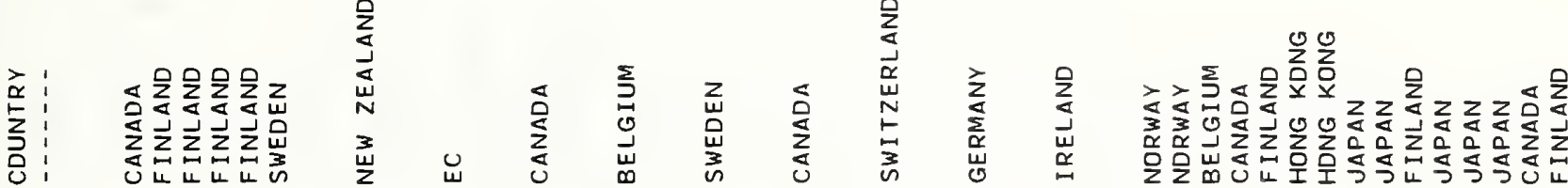

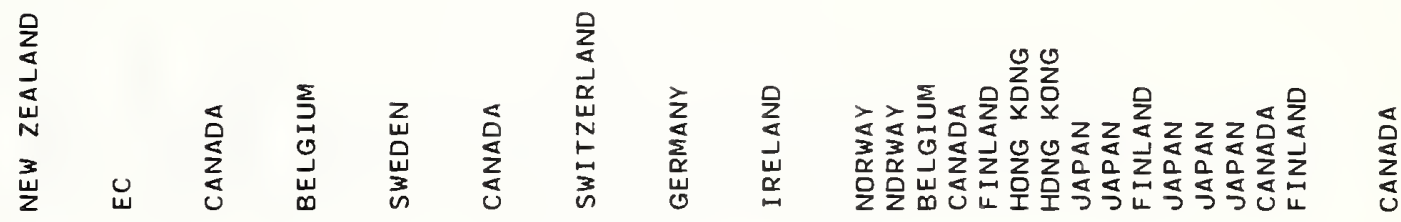

氛:
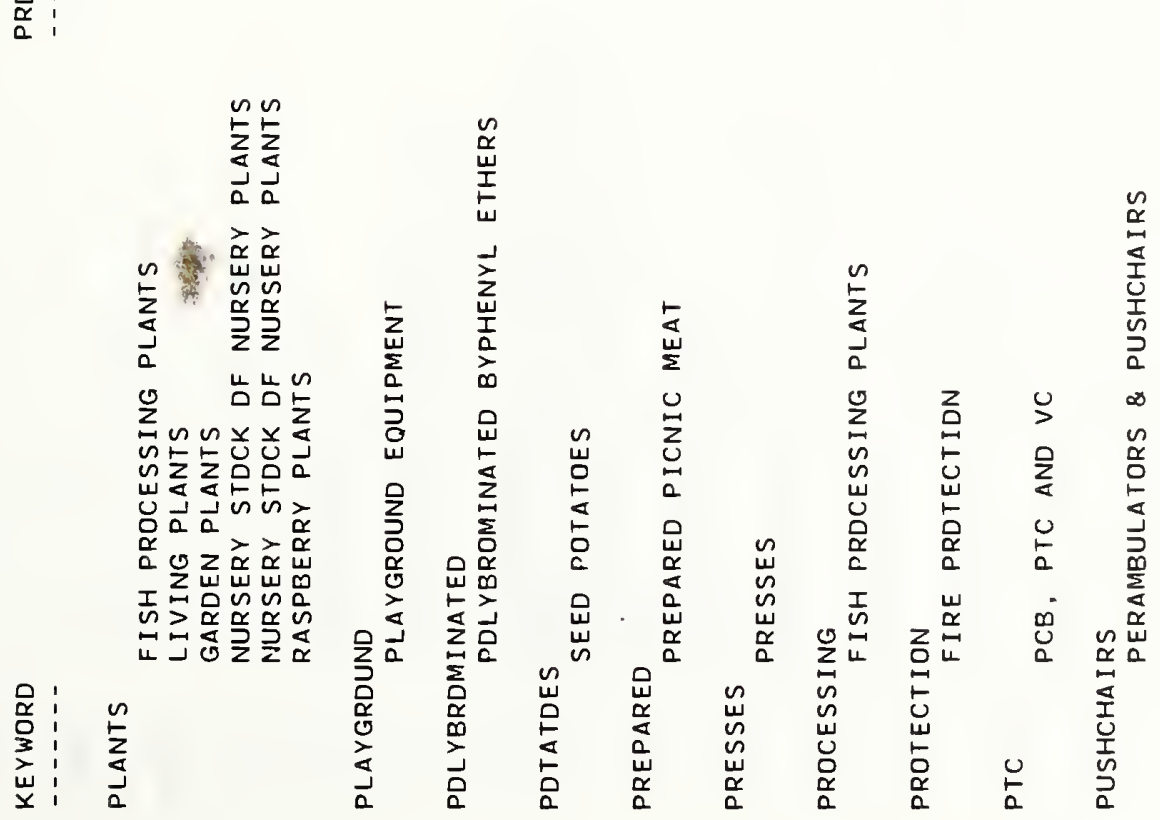

츨

岗

$00 n$

菒品

唩

衣号

岁㱐

造 


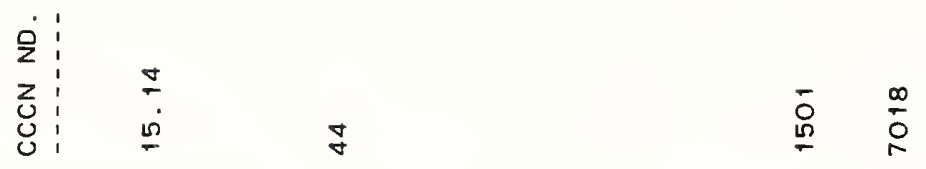

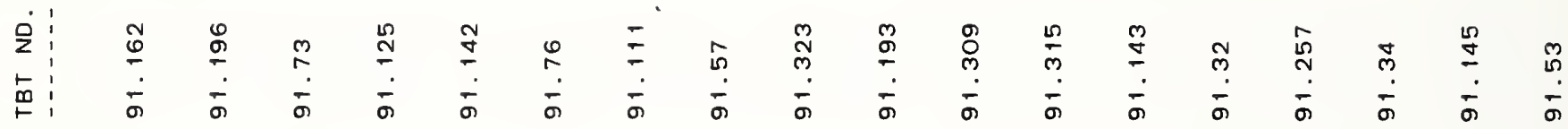

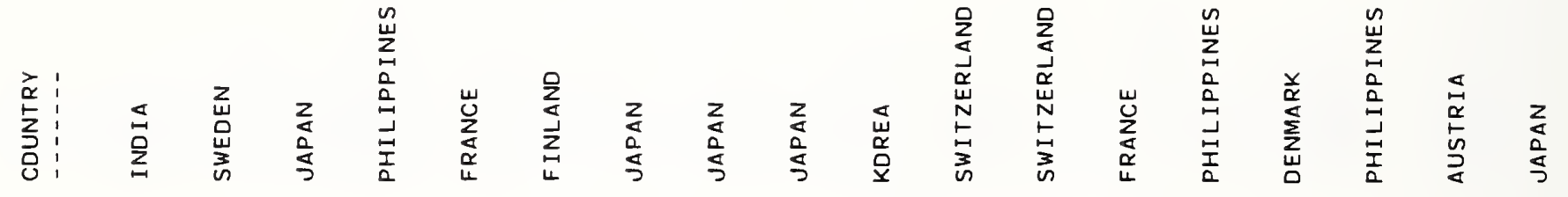

:

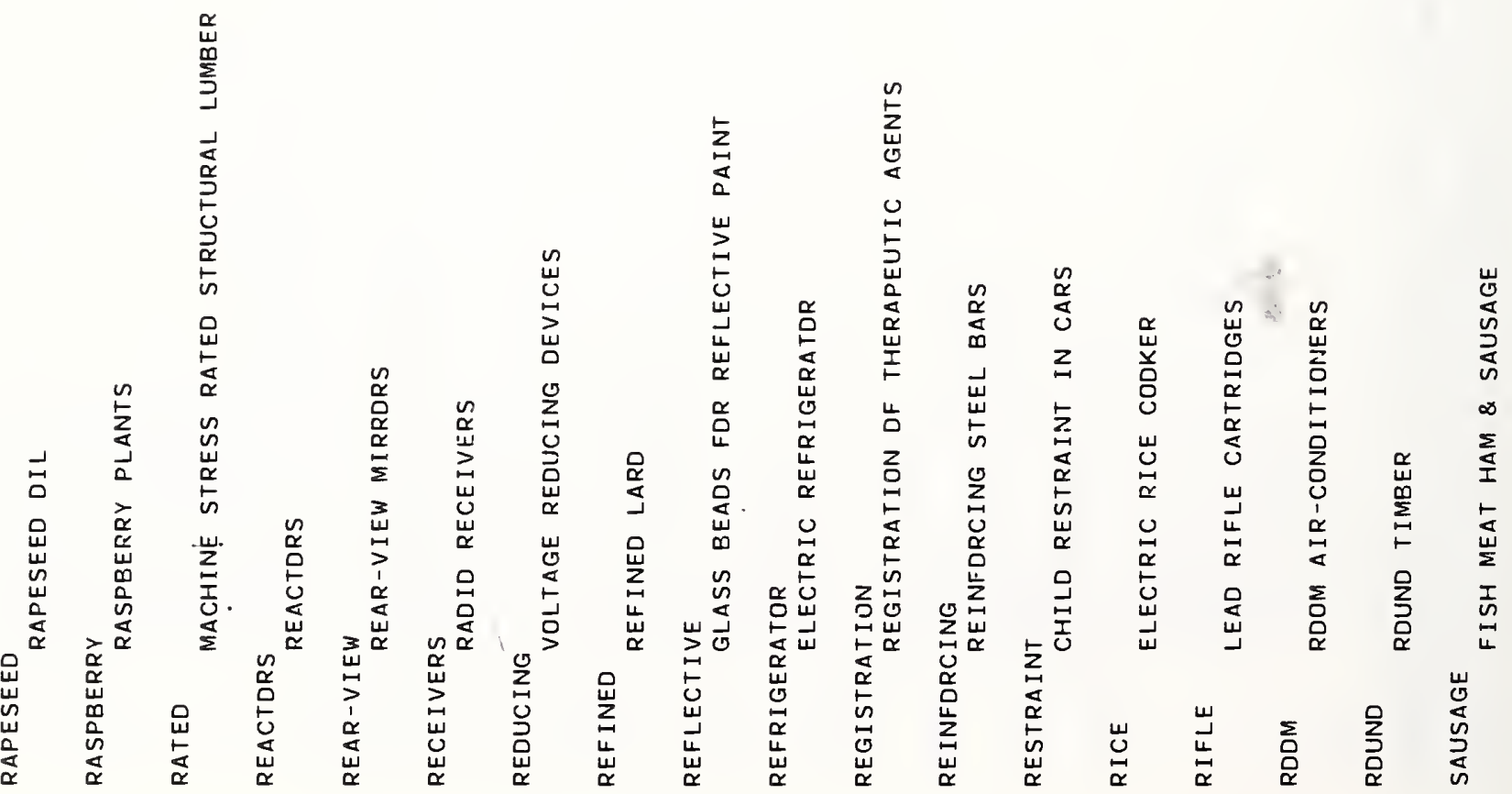




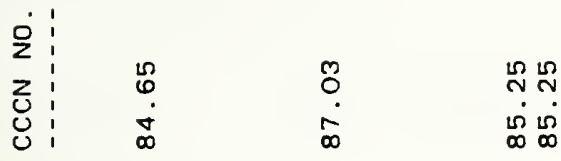

$\stackrel{2}{=} \quad \stackrel{0}{0}$

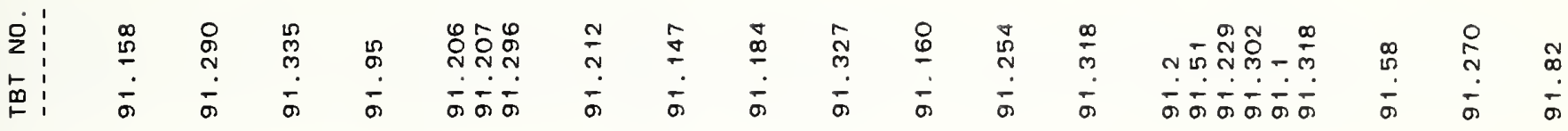
㐫

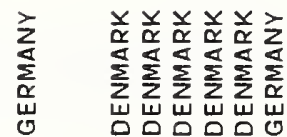

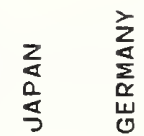

$\frac{2}{2}$

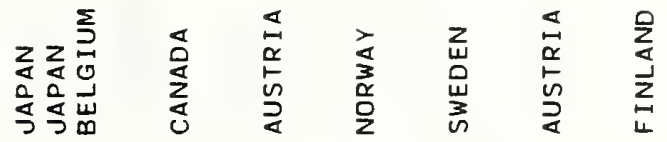

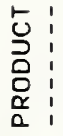

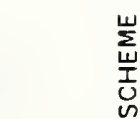

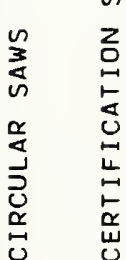

总

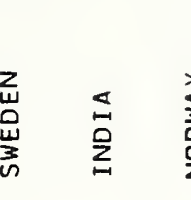

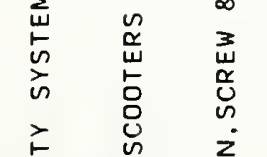

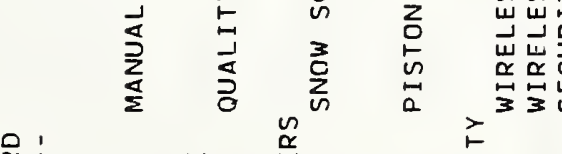

Ư

岩

己る

品 $\frac{N}{1}$

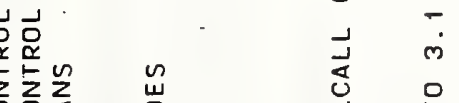

点出

觜息

岕岕范

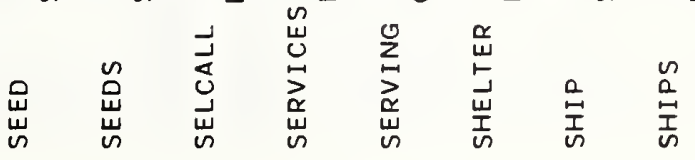

立

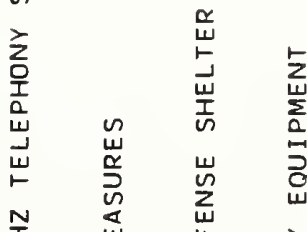

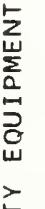

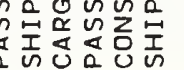

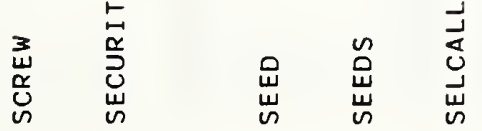




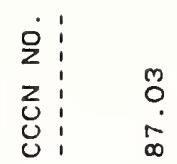

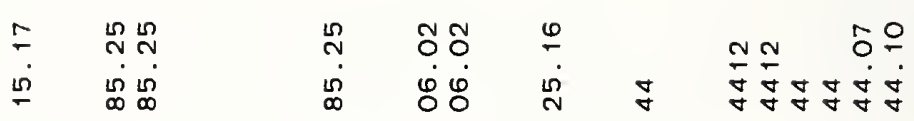

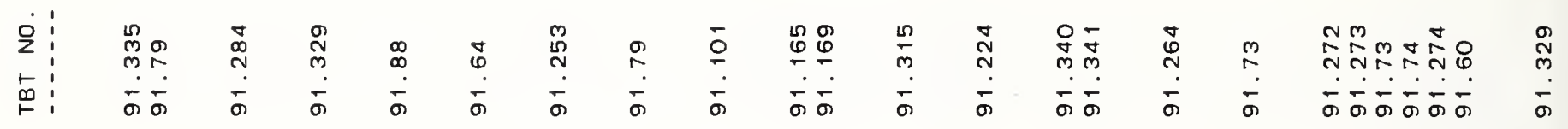

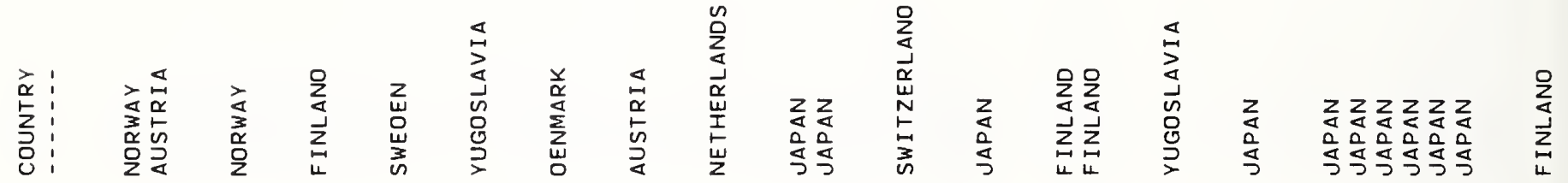

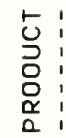

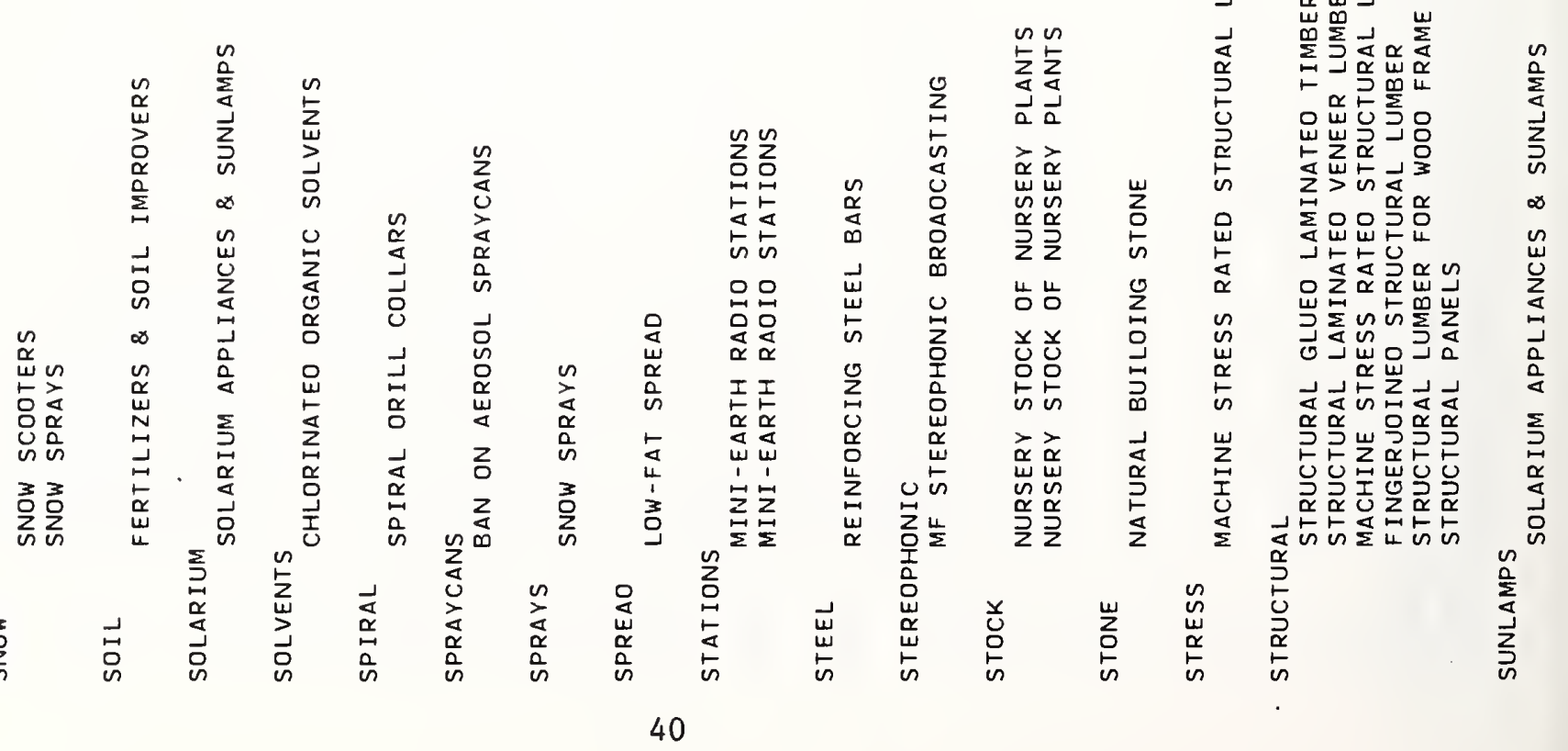




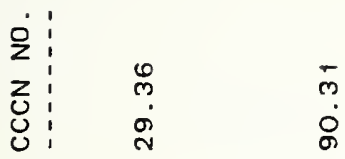

$=\frac{1}{\infty}$

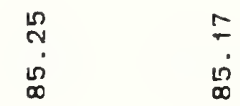

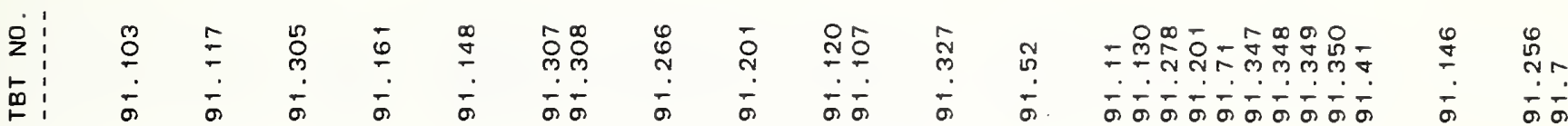

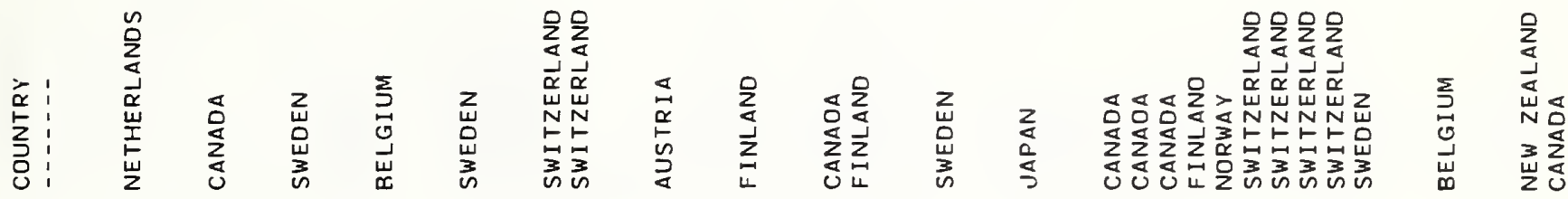

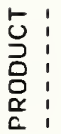

点
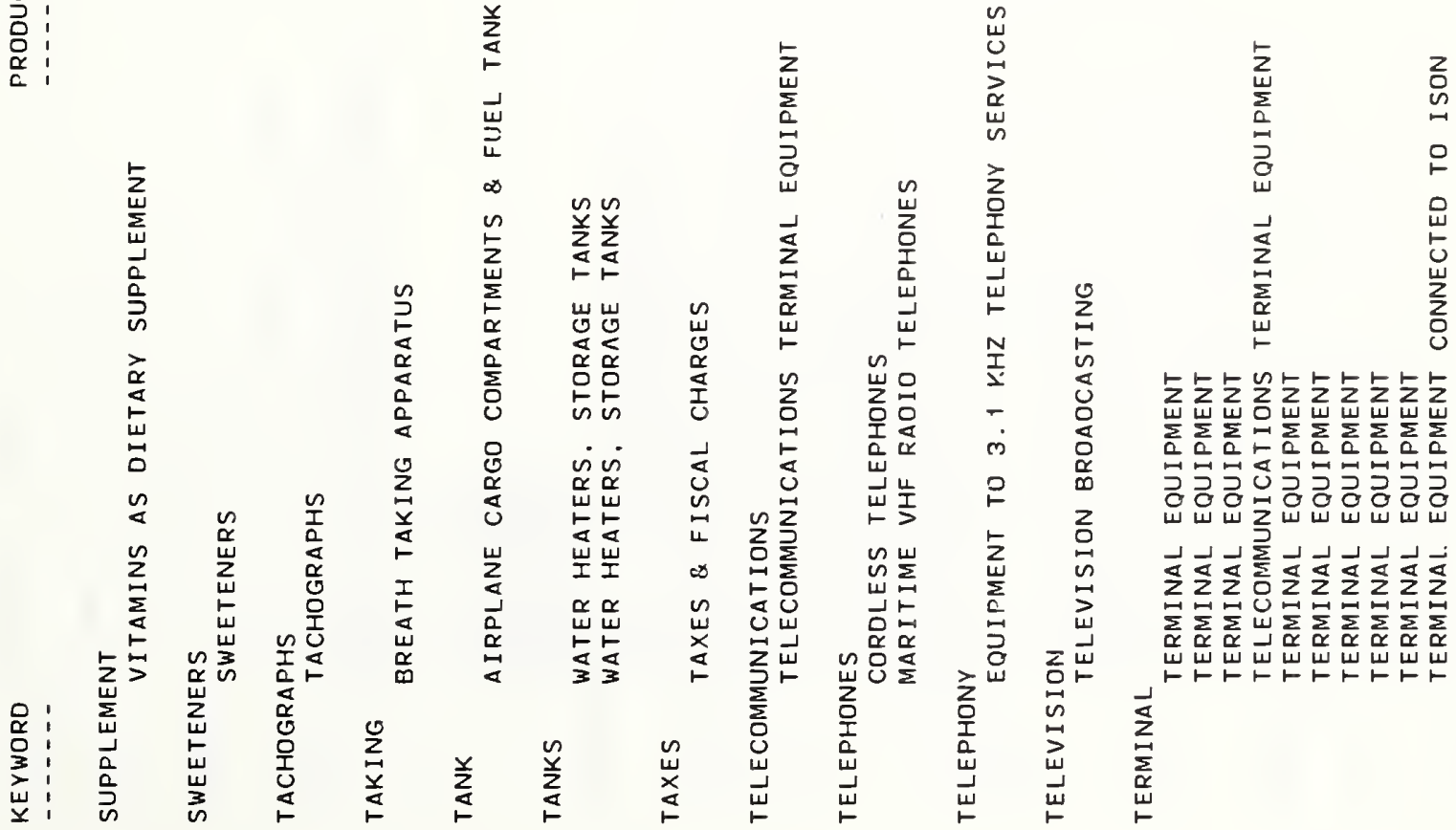

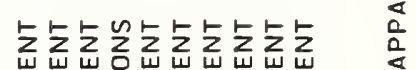

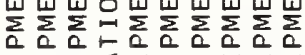

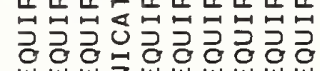

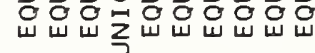

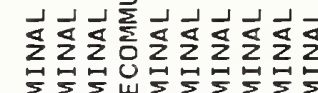

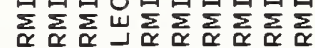

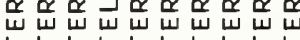

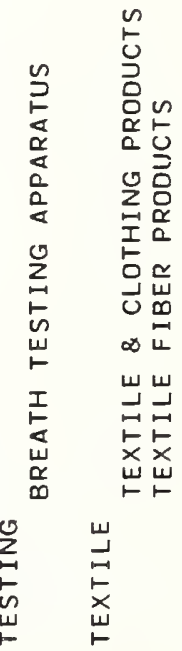




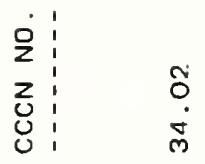

$\frac{d}{d}$

مิ

N

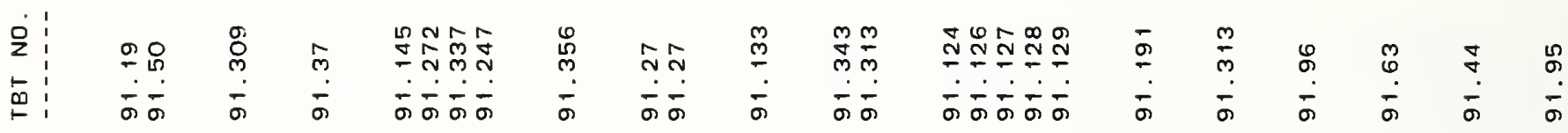

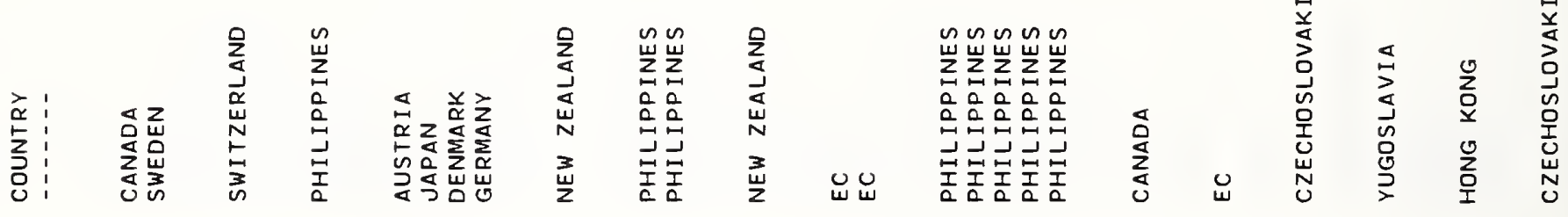

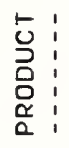

논

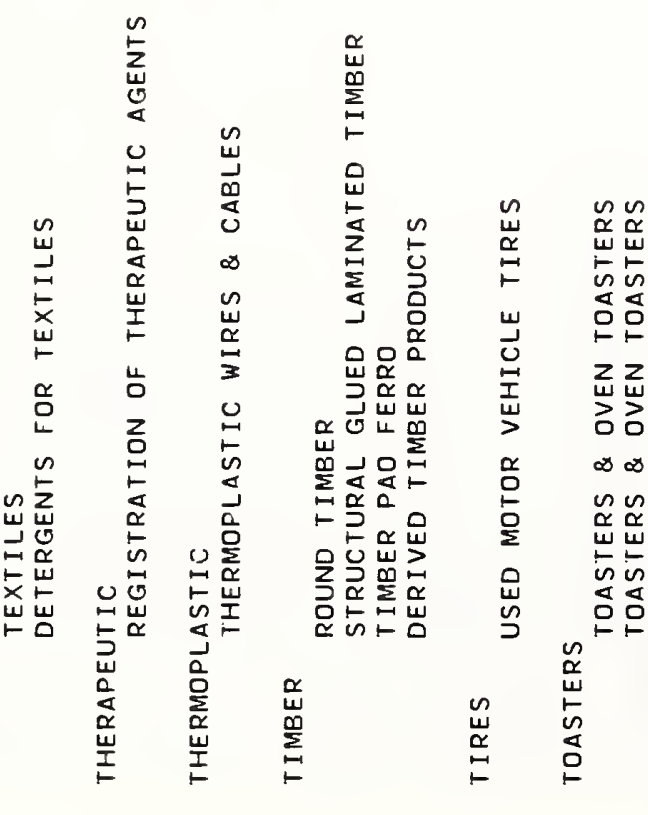

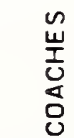

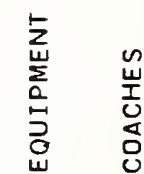

范文

$\begin{array}{lll}2 & 0 \\ 0 & 0\end{array}$

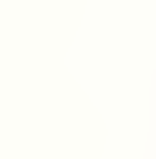

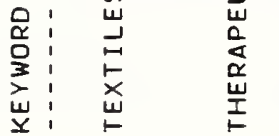

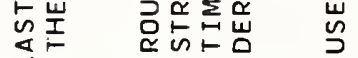

a
5

点

$\overbrace{\infty}^{\infty}$

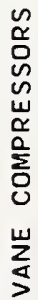

崖剀

$\infty \dot{\sim}$

吕

nosing

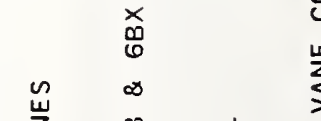

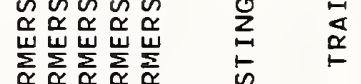

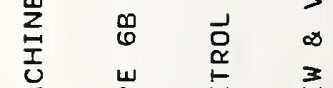

它品

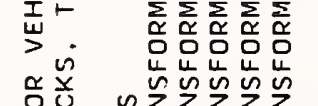

$\sum_{1}^{0}$

峁

点 


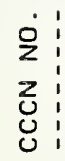

$\stackrel{1}{\circ}$

Non

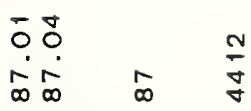

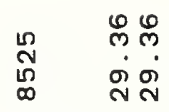

$\begin{array}{ll}\frac{1}{m} & \stackrel{\text { ก }}{n}\end{array}$

$\stackrel{n}{\stackrel{n}{\infty}}$

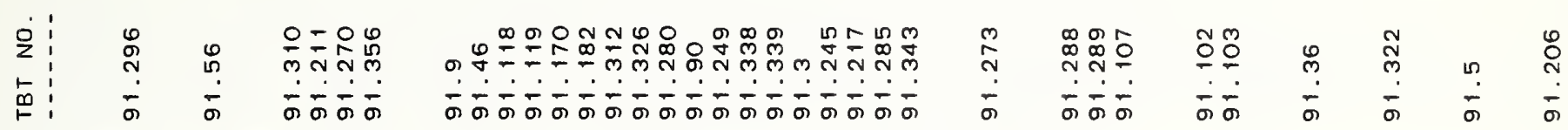

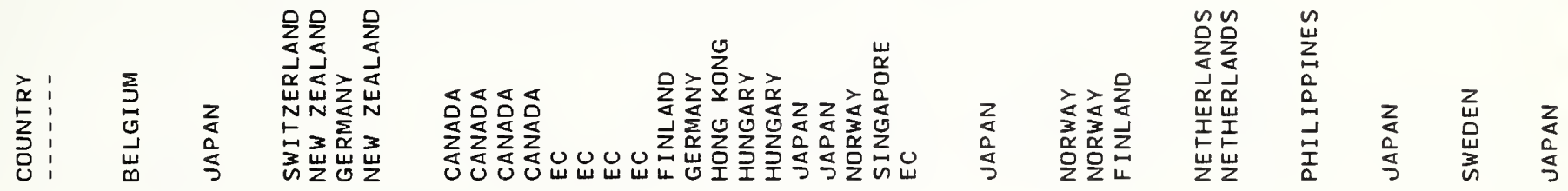

\begin{tabular}{l:}
5 \\
\hdashline \\
\hdashline \\
0 \\
$\alpha$ \\
$\alpha$
\end{tabular}

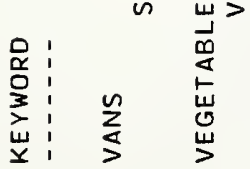

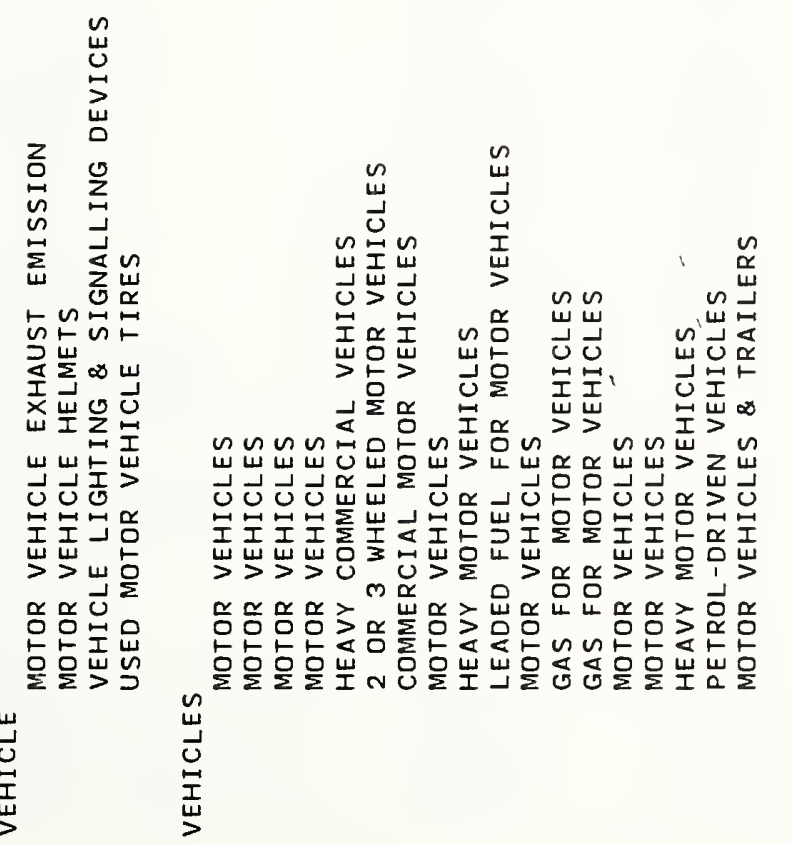

号号

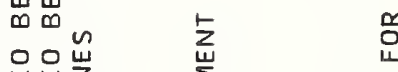

运定定

$\propto \square$ 연

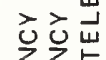

천

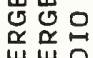

崖崖是

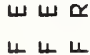

殅殅崖

岂崖崖

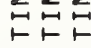

采采金

$\sum_{\Sigma} \sum_{\Sigma} \sum_{\Sigma}$

殅 


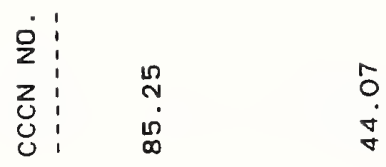

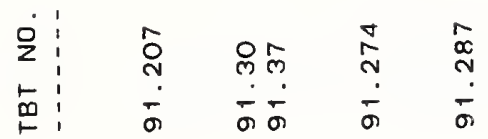

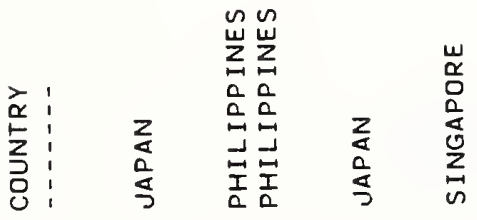

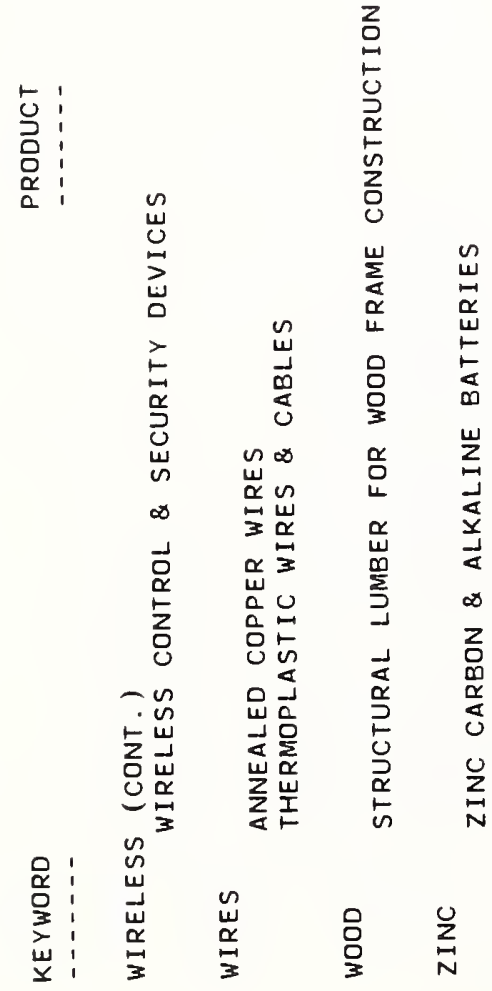


Thirty of the 40 signatories to the standards code contacted the National Center for standards and Certification Information for information on proposed or existing U.S. standards and regulations. The number in parentheses () indicates the number of inquiries which came from the GATT inquiry points. Many requests come from private companies or individuals.

Party Receiving Inquiries

United States

\section{NUMBER OF INQUIRIES IN 1991}

\section{Inquiries originating In}

Total

Austria

Belgium

Brazil

Canada

Czechoslovakia

Denmark

Egypt

Finland

France

Germany

Hong Kong

Hungary

India

Ireland

Israel

Italy

Japan

Mexico

Netherlands

New Zealand

Norway

Philippines

Portugal

Singapore

spain

Sweden

Switzerland

Tunisia

United Kingdom

Yugoslavia

\begin{tabular}{|c|c|c|c|c|c|}
\hline \multicolumn{2}{|c|}{$\begin{array}{l}\text { Total } \\
\text { (of which } \\
\text { from respective) } \\
\text { Inquiry Points) }\end{array}$} & \multicolumn{2}{|c|}{$\begin{array}{l}\text { Regarding } \\
\text { Proposed } \\
\text { Rules and } \\
\text { Regulations }\end{array}$} & \multicolumn{2}{|c|}{$\begin{array}{l}\text { Regarding } \\
\text { Existing } \\
\text { Rules and } \\
\text { Regulations }\end{array}$} \\
\hline Rec'd & Answ'd & Rec'd & Answ'd & Rec'd & Answ'd \\
\hline $257(161)$ & $\underline{257(161)}$ & $\underline{125}$ & $\underline{125}$ & $\underline{132}$ & $\underline{132}$ \\
\hline $2(1)$ & $2(1)$ & 2 & 2 & & \\
\hline $4(2)$ & $4(2)$ & 1 & 1 & 3 & 3 \\
\hline $6(4)$ & $6(4)$ & & & 6 & 6 \\
\hline $17(2)$ & $17(2)$ & 1 & 1 & 16 & 16 \\
\hline $6(6)$ & $6(6)$ & 6 & 6 & & \\
\hline $19(17)$ & 19 (17) & 1 & 1 & 18 & 18 \\
\hline 2 & 2 & & & 2 & 2 \\
\hline $11(11)$ & $11(11)$ & 3 & 3 & 8 & 8 \\
\hline 21 (19) & 21 (19) & 13 & 13 & 8 & 8 \\
\hline 6 & 6 & 1 & 1 & 5 & 5 \\
\hline $11(6)$ & $11(6)$ & 4 & 4 & 7 & 7 \\
\hline $4(4)$ & $4(4)$ & 4 & 4 & & \\
\hline $23(18)$ & $23(18)$ & 18 & 18 & 5 & 5 \\
\hline $4(1)$ & $4(1)$ & 1 & 1 & 3 & 3 \\
\hline $5(2)$ & $5(2)$ & & & 5 & 5 \\
\hline 4 & 4 & & & 4 & 4 \\
\hline $14(1)$ & $14(1)$ & 8 & 8 & 6 & 6 \\
\hline $9(3)$ & $9(3)$ & & & 9 & 9 \\
\hline 2 & 2 & & & 2 & 2 \\
\hline $9(7)$ & $9(7)$ & 5 & 5 & 4 & 4 \\
\hline 1 & 1 & & & 1 & 1 \\
\hline $8(8)$ & $8(8)$ & 7 & 7 & 1 & 1 \\
\hline 1 & 1 & & & 1 & 1 \\
\hline 1 & 1 & 1 & 1 & & \\
\hline $2(1)$ & $2(1)$ & 1 & 1 & 1 & 1 \\
\hline $19(17)$ & $19(17)$ & 15 & 15 & 4 & 4 \\
\hline $2(1)$ & $2(1)$ & 2 & 2 & & \\
\hline $3(3)$ & $3(3)$ & 3 & 3 & & \\
\hline $38(26)$ & $38(26)$ & 27 & 27 & 11 & 11 \\
\hline $3(1)$ & $3(1)$ & 1 & 1 & 2 & 2 \\
\hline
\end{tabular}





\begin{tabular}{|c|c|}
\hline $\begin{array}{l}\text { U.S. DEPARTMENT OF COMMERCE } \\
\text { NATIONAL INSTITUTE OF STANDARDS AND TECHNOLOGY }\end{array}$ & 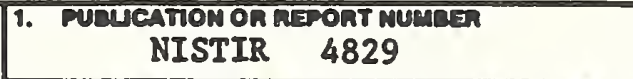 \\
\hline \multirow{3}{*}{$\begin{array}{l}\text { NATIONAL INSTITUTE OF STANDARDS AND TECHNOLOGY } \\
\text { BIBLIOGRAPHIC DATA SHEET }\end{array}$} & 2 PERPORMIMO ORQANRATION AEPOAT MUMLES \\
\hline & \\
\hline & $\begin{array}{l}\text { 3. FUQWCATION DATE } \\
\text { ApI11 } 1992 .\end{array}$ \\
\hline \multicolumn{2}{|l|}{$\begin{array}{l}\text { 4. TILE AND SULTILI } \\
\text { GATT STANDARDS CODE ACTIVITIES OF THE NATIONAL INSTITUTE OF STANDARDS } \\
\text { AND TECHNOLOGY } 1991\end{array}$} \\
\hline \multicolumn{2}{|l|}{ JoAnne R.Overman } \\
\hline \multirow{2}{*}{ 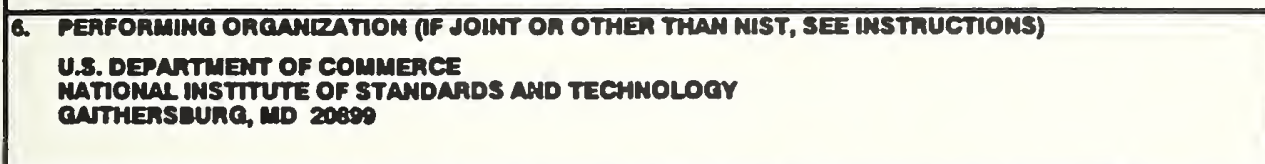 } & 7. CONTRACT/ORATT MUMEER \\
\hline & $\begin{array}{l}\text { Q. TYPE OF REPOAT AMD PERIOD COVERED } \\
\text { Annual, } 1991\end{array}$ \\
\hline
\end{tabular}

10. SUPPLEMENTAAYMOTES

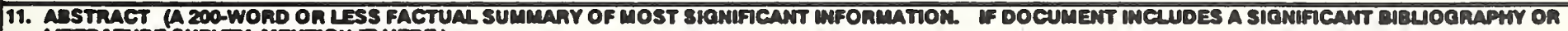
UTERATUAE SURVI, MENTION TT HERE)

Th1s report describes the GATT Standards Code activities conducted by the Standards Code and Information Program, National Institute of Standards and Technology(NIST), for calendar year 1991. NIST responsibilities Include operating the O.S. GATT Inquiry point for Information on standards and certification activities; notifying the GATT Secretariat of proposed U.S. Federal Government standards-based rules that might significantly affect trade; assisting U.S. Industry with standards-related trade problems; and responding to Inquiries about proposed forelgn and D.S. regulations.

12. KEY WOADS (6 TO 12 EMTRIES; APHABETICAL OADEA; CAPTALIE ORLY PROPEA MAMES; AMD SEPARATE KEY WOADS DY SEMICOLOHS)

certification information; GATT Standards Code; notifications; proposed forelgn regulations; standards information; technical assistance

13. AVALABIUTY

$\mathrm{X}$ Unumited

FOR OFFICKL OISThIBUTION. DO NOT RESASE TO MATIOMAL TECHNICNL MFOAMATION SEAVCE (NTIS).

ONDEA FROM SUPERINTENDENT OF DOCUMENTS, U.S. COVENHMENT PAINTINO OFFICE, WASHMOTON, DC 20402

OMDEA FROM MATIOMAL TECHNICAL INFOAMATION SERVICE (WTS), SPAIMOFED, VA 2161.

14. MULBEER OF PRINTED PAQES

15. PAICE 
• 

